\title{
Closed-loop stimulation of the medial septum terminates epileptic seizures
}

\author{
(D)Yuichi Takeuchi, ',2,3 (D) Márk Harangozó,' Lizeth Pedraza, I,3 (D)Tamás Földi,, 1,3 \\ Gábor Kozák,' (DQun Li ${ }^{1,3}$ and (DAntal Berényi ${ }^{1,3,4,5}$
}

See Magloire and Lignani (doi.10.1093/brain/awab051) for a scientific commentary on this article.

Temporal lobe epilepsy with distributed hippocampal seizure foci is often intractable and its secondary generalization might lead to sudden death. Early termination through spatially extensive hippocampal intervention is not feasible directly, because of the large size and irregular shape of the hippocampus. In contrast, the medial septum is a promising target to govern hippocampal oscillations through its divergent connections to both hippocampi. Combining this 'proxy intervention' concept and precisely timed stimulation, we report here that closed-loop medial septum electrical stimulation can quickly terminate intrahippocampal seizures and suppress secondary generalization in a rat kindling model. Precise stimulus timing governed by internal seizure rhythms was essential. Cell type-specific stimulation revealed that the precisely timed activation of medial septum GABAergic neurons underlaid the effects. Our concept of time-targeted proxy stimulation for intervening pathological oscillations can be extrapolated to other neurological and psychiatric disorders, and has potential for clinical translation.

1 MTA-SZTE ‘Momentum’ Oscillatory Neuronal Networks Research Group, Department of Physiology, University of Szeged, Szeged 6720, Hungary

2 Department of Neuropharmacology, Graduate School of Pharmaceutical Sciences, Nagoya City University, Nagoya, 467-8603, Japan

3 Neurocybernetics Excellence Center, University of Szeged, Szeged 6720, Hungary

4 HCEMM-USZ Magnetotherapeutics Research Group, University of Szeged, Szeged 6720, Hungary

5 Neuroscience Institute, New York University, New York, NY 10016, USA

Correspondence to: Antal Berényi

MTA-SZTE ‘Momentum’ Oscillatory Neuronal Networks Research Group, Department of Physiology

University of Szeged, 10 Dom sqr., Szeged 6720, Hungary

E-mail: drberenyi@gmail.com

Keywords: epilepsy; closed-loop; medial septum; deep brain stimulation; optogenetics

Abbreviations: $\mathrm{Ctx}=$ neocortex $; \mathrm{DBS}=\mathrm{deep}$ brain stimulation; $\mathrm{EC}=$ entorhinal cortex; E-stim = electrical stimulation; $\mathrm{HPC}=$ hippocampus; $\mathrm{LFP}=$ local field potential; $\mathrm{MI}=$ modulation index; TLE = temporal lobe epilepsy

\section{Introduction}

Approximately $1 \%$ of the world population has epilepsy and $30 \%$ of these cases are refractory (Kwan et al., 2011; Chen et al., 2018). Temporal lobe epilepsy (TLE) is often drug-resistant and uncontrolled generalized seizures increase the risk of sudden unexpected death in epilepsy (Bone et al., 2012; Massey et al., 2014). Although effective, surgical resection of the seizure focus is highly invasive, irreversible, and frequently associated with cognitive dysfunction. In addition, it may not be the best solution for patients with ambiguous or multifocus bilateral TLE (Berg et al., 2003; 
Holmes et al., 2003; Selwa et al., 2003). Thus, a new therapeutic approach is desirable. Deep brain stimulation (DBS) has been investigated to treat neurological and psychiatric disorders including epilepsy ( $\mathrm{Li}$ and Cook, 2018). Direct stimulation of the hippocampus (HPC) can reduce seizure occurrence, especially in medial TLE (Han et al., 2014; Li and Cook, 2018). However, because the HPC is a relatively large bilateral structure, direct electrical stimulation may affect only a small portion of the seizure-generating structure (Cukiert and Lehtimäki, 2017). An alternative therapeutic approach is stimulation of another brain region rather than the seizure focus region (Fisher et al., 2010; Fountas et al., 2010; Paz and Huguenard, 2015).

Here, we propose that HPC activity can be thoroughly controlled in a technically feasible way by interfering with a smaller circumscribed structure, which has massively divergent efferent connections to the whole extent of both HPCs (proxy intervention). The medial septum is an attractive target of such proxy intervention because it is a small midline structure with diverging projections onto both HPCs (Dutar et al., 1995). The septohippocampal pathway plays an important role in governing theta (Buzsáki, 2002; Kang et al., 2017) and high-frequency HPC oscillations (Vandecasteele et al., 2014); the latter are particularly vulnerable to turn into seizure activity (Malow et al., 1999; Ewell et al., 2015; Moxon et al., 2019).

Clinical brain stimulation technologies, being either nonresponsive (continuous) or responsive (i.e. triggered by the detection of certain activity patterns), deliver hard-coded, nonadaptive sequences of preset pulse trains or random noise waveforms (Morrell, 2011). Although these open-loop approaches are effective in reducing seizure occurrence by decreasing seizure susceptibility, they cannot quickly terminate already initiated seizures (Meiron et al., 2019). This would require temporally aligned stimulus delivery targeting specific phases of pathological oscillations (Berényi et al., 2012; Dejean et al., 2016; Kozák and Berényi, 2017). Because of its direct reciprocal connections, the septohippocampal axis resonates in a highly coherent manner in both physiological and pathophysiological conditions, including ictal periods of epilepsy (Sinel'nikova et al., 2009; Tsanov, 2018). The stimulation of medial septum neurons can precisely entrain HPC local field potentials (LFPs) (Fuhrmann et al., 2015; Robinson et al., 2016; Zutshi et al., 2018). In addition, the medial septum can powerfully gate oscillations not only in the HPC but also in the entorhinal cortex (EC), which is the bottleneck structure for seizure propagation from the HPC to the neocortex (Ctx) (McIntyre and Gilby, 2008). Given these anatomical and temporal features of the septohippocampal axis, we hypothesized that the medial septum can be a powerful seizure regulation centre for on-demand control of TLE. Thus, we implemented a closed-loop stimulation of the medial septum driven by seizure patterns, and investigated whether time-targeted precise stimulus delivery to the medial septum can cease already initiated HPC seizures, before they generalize.

The present study was designed to obtain a mechanistic understanding of possible seizure terminating effects of the medial septum in a cell-type specific manner. The medial septum has GABAergic, glutamatergic and cholinergic neuron populations. All of these populations project to distinct targets of the HPC and the EC (Colom et al., 2005; Joshi et al., 2017; Desikan et al., 2018). They have distinct excitatory or inhibitory postsynaptic effects on these targets (Chamberland et al., 2010; Robinson et al., 2016; Desikan et al., 2018). Optogenetics has been successfully applied to medial septum populations in dissecting their physiological roles in the septohippocampal axis (Vandecasteele et al., 2014; Robinson et al., 2016; Zutshi et al., 2018). We used selective optogenetic effectors in our closed-loop approach to dissect the hypothesized seizure-terminating effects.

We found that seizure rhythm-congruent medial septum electrical stimulation can effectively terminate HPC seizures in their early phases and suppress secondary generalizations. Responsive, but open-loop (fixed pattern) medial septum electrical stimulation did not terminate the seizures. This finding emphasized that precise seizure rhythm-matched stimulus delivery is essential for breaking ongoing pathological oscillations. Time-targeted optogenetic experiments revealed that precisely timed activation of medial septum GABAergic neurons mainly underlaid the seizure termination effect, while the pre-ictal activation of medial septum cholinergic neurons reduced seizure susceptibility.

\section{Materials and methods}

\section{Rat models}

All experiments were performed in accordance with European Union guidelines (2003/65/CE) and the National Institutes of Health Guidelines for the Care and Use of Animals for Experimental Procedures. The experimental protocols were approved by the Ethical Committee for Animal Research at the Albert Szent-Györgyi Medical and Pharmaceutical Center of the University of Szeged (XIV/218/2016). Fifty adult LongEvans rats of both sexes (eight wild-type and 42 transgenic, 36 months old, 300-640 g) were used in the present study. VGAT-Cre [LE-Slc32a1 ${ }^{\text {tm1 (cre)Sage }}$; RGD Cat\# 12905033; RRID: RGD_12905033] and CaMKII $\alpha$-Cre rats [LECamk2atm1(IRES-cre)Sage, RGD Cat\# 12905032; RRID: RGD_12905032] were purchased and licensed from Horizon Discovery. ChAT-Cre rats [LE-Tg(ChAT-Cre) 5.1Deis, RGD Cat\# 10401204; RRID: RGD_10401204] were provided by Dr Deisseroth (Witten et al., 2011). The rats were fed a commercial diet, provided with water ad libitum and housed as groups in an environment with controlled temperature and lighting (12/12 h light/dark cycle). They were either bred from in-house colonies or purchased from Charles River. No animals were excluded from our analysis.

\section{Genotyping}

ChAT-Cre rats were maintained as male heterozygotes and genotyped by a standard PCR procedure with the following primers: CW-Cre2, 5'-ACC TGA TGG ACA TGT TCA GGG ATC G-3' and CW-Cre3, 5'-TCC GGT TAT TCA ACT TGC 
ACC ATG C-3', producing 108-bp fragments from the cre allele (Iwasato et al., 2004). Other Cre driver lines were maintained as homozygotes of both sexes after initial genotyping using the manufacturer's PCR protocols. No randomization or blinding was used for the experiments outlined below.

\section{Stereotaxic viral vector injections}

For channelrhodopsin 2 (ChR2) expression, AAV5-hSynhChR2(H134R)-mCherry or AAV5-EF1 $\alpha$-DIOhChR2(H134R)-mCherry (UNC Vector Core) was injected into the medial septum of wild-type or Cre-driver animals, respectively, using a standard intracranial virus vector injection technique. Briefly, animals were anaesthetized with $1-3 \%$ isoflurane and then mounted on a stereotaxic apparatus. Atropine $(0.1$ $\mathrm{mg} / \mathrm{kg}$, s.c.) was administered immediately after the anaesthesia induction. Stages of anaesthesia were maintained by confirming the lack of nociceptive reflex. The rectal temperature was maintained at $36-37^{\circ} \mathrm{C}$ with a DC temperature controller (TMP-5b; Supertech). The head position was adjusted so that the bregma and lambda were at the same level. A small incision on the skin was then made for craniotomy after subcutaneous lidocaine injections. A craniotomy was drilled at $1.0 \mathrm{~mm}$ to the left of the midline and $0.5 \mathrm{~mm}$ anterior from the bregma. A small incision on the dura mater was then made. A glass capillary (tip 10-20 $\mu \mathrm{m})$ filled with an adeno-associated virus (AAV) vector without dilution (qPCR titre: $3-5 \times 10^{12} \mathrm{vg} / \mathrm{ml}$ ) was installed with an auto-nanolitre injector (Nanoject II; Drummond Scientific). The injector was $9.5^{\circ}$ left of the sagittal plane to avoid the sagittal sinus. Three penetrations were made from the following stereotaxic coordinates: $0.2,0.5$ and $0.8 \mathrm{~mm}$ anterior from the bregma and $1.0 \mathrm{~mm}$ left of the midline. Along each penetration, $0.3 \mu \mathrm{l}$ of viral solution was ejected at 5.8, 6.1 and $6.4 \mathrm{~mm}$ from the pia at a rate of $0.9 \mathrm{nl} / \mathrm{s}$. The capillary tip was kept there for at least $5 \mathrm{~min}$ after each ejection and then gently retracted. The cranial window was covered with a haemostatic gel, and the skin wound was then sutured. The injected animals were housed at least 4 weeks prior to optogenetic intervention.

\section{Electrophysiology}

To monitor electrographic seizures, intracranial LFP recordings were made from freely-moving animals. Intra-medial septum electrophysiological correlates of optogenetic stimulation were confirmed in anaesthetized animals.

\section{Construction of recording electrodes}

Tripolar tungsten wire electrodes were prepared as previously reported (Kozák et al., 2018). Briefly, we glued three $50 \mu \mathrm{m}$ diameter HML-insulated tungsten wires (Tungsten 99.95\%; California Fine Wire) into a $25 \mathrm{G}$ stainless-steel tube. Tips of the tungsten wires were spaced at a $0.4 \mathrm{~mm}$ axial distance. The impedance of each wire was $30-90 \mathrm{k} \Omega$ at $1 \mathrm{kHz}$.

\section{Chronic implantation surgery}

Each animal was implanted with 10 tripolar electrodes under stereotaxic guidance as previously described (Kozák et al., 2018). In total, 30 recording sites were distributed to the bilateral dorsal hippocampus (rHPC, lHPC), right medial entorhinal cortex (MEC) and right somatomotor cortex (S1/M1). The stereotaxic coordinates of recording sites were: S1/M1 (AP: 1.0 and $2.5 \mathrm{~mm}$ anterior from the bregma; ML: $3.0 \mathrm{~mm}$; DV: 1.2 ,
1.6 and $2.0 \mathrm{~mm}$ from the dura), HPC (AP: 3.5, 4.5 and $5.5 \mathrm{~mm}$ posterior from the bregma and ML: $2.0,3.0$ and $4.0 \mathrm{~mm}$, respectively; DV: 2.6, 2.9 and $3.3 \mathrm{~mm}$ from the dura), MEC (AP: penetrated from the $0.1 \mathrm{~mm}$ anterior from the transverse sinus at a $20^{\circ}$ caudally tilted angle from the coronal plane; ML, 3.5 and $4.5 \mathrm{~mm}$; distance: $3.2,3.6$ and $4.0 \mathrm{~mm}$ from the dura). The dura mater was not removed but penetrated with tripolar electrodes. After insertion, the electrodes were physically affixed with a dental cement (Unifast Trad). Two stainless-steel machine screws were installed in the skull above the cerebellum as reference and ground electrodes. A bipolar stimulus electrode was placed targeting the HPC commissure for kindling and seizure induction during the experiments (see below). Either an optical cannula or a bipolar stimulus electrode was also inserted into the medial septum. Finally, an on-head Faraday cage made of copper mesh connected to the ground electrode was cemented on the skull to both prevent environmental noise and to physically protect the implanted components. The implanted animals were housed in individual cages.

\section{Data acquisition from freely moving animals}

Daily LFP recordings were performed in the animals' own home cages. The tungsten wire electrodes were connected to a signal multiplexing headstage (HS3_v1.3, Amplipex) attached to a thin and light cable (36 AWG Soveron served Litz wire; KerriganLewis, Alpha wire) pending from a trolley system on the room ceiling. This allowed for the free movement of the animals. To avoid any twisting and over-tension of the recording cables and to provide an optical fibre for optogenetic stimulation, a borethrough electrical commutator (VSR-TC-15-12; Victory-Way Electronic) and an optical rotary joint (RJPSF2; Thorlabs) were integrated into the recording cables. The multiplexed signals were acquired at $500 \mathrm{~Hz}$ per channel for closed-loop intervention experiments (Kozák and Berényi, 2017). Signals at $20 \mathrm{kHz}$ per channel were acquired for open-loop intervention experiments and other recordings (KJE-1001, Amplipex) (Berényi et al., 2014).

\section{Post-mortem localization of the recording sites}

After data acquisition, animals were deeply anaesthetized with $1.5 \mathrm{~g} / \mathrm{kg}$ urethane (i.p.). One or two recording sites of each tripolar electrode were lesioned with $100 \mu \mathrm{A}$ anodal direct current for $10 \mathrm{~s}$ via tips. The animals were transcardially perfused and their brains were sectioned as described below. The sections were stained with $1 \mu \mathrm{g} / \mathrm{ml}$ DAPI in distilled water (D8417; Sigma-Aldrich), coverslipped and observed with a confocal microscopy (Supplementary Fig. 1).

\section{Hippocampal electrical kindling}

\section{Kindling procedure}

A bipolar stimulus electrode for kindling and seizure induction was prepared using the same tungsten wire as tripolar recording electrodes. Tips of the bipolar stimulus electrodes were axially spaced $0.5 \mathrm{~mm}$ apart and insulations $0.4-0.5 \mathrm{~mm}$ around the tips were stripped to decrease their impedance to $10-20 \mathrm{k} \Omega$ at 1 $\mathrm{kHz}$. The stimulus electrode was implanted on the HPC commissure during the chronic implantation surgery for recording electrodes in the following stereotaxic coordinate: AP, $0.84 \mathrm{~mm}$ posterior from the bregma; ML, penetrated from $1.5 \mathrm{~mm}$ right of the midline at an $18^{\circ}$ right-tilted angle from the parasagittal 
plane; distance, $4.5 \mathrm{~mm}$ from the dura. The kindling electrode was fixed with dental cement. After recovery from the implantation surgery, the HPC was stimulated daily at subconvulsion intensity via the kindling electrode. Each kindling stimulation consisted of $120 \times 0.5 \mathrm{~ms}$ positive $-0.5 \mathrm{~ms}$ negative bipolar rectangular pulses at $62.5 \mathrm{~Hz}$ (Gelinas et al., 2016) generated via a programable isolator in current control mode (STG4008; Multi Channel Systems). Stimulus intensity was determined as the minimum that induced after-discharge $(10-25 \mathrm{~Hz}$ population spikes synchronously observed in $>50 \%$ of HPC channels after the HPC commissure stimulation) in HPC LFPs, which was typically $\pm 20-200 \mu \mathrm{A}$. The kindling stimulation was conducted six times per day for 10 days. Each stimulation was delivered with at least 30-min interstimulus intervals to develop secondary generalized seizures in response (Supplementary Fig. 2).

\section{Behavioural monitoring}

The behaviour of animals was continuously monitored during the electrophysiological recordings with a webcam (LifeCam Studio) in VGA resolution with a 30-fps rate and synchronized with the acquired neuronal data. The severity of motor seizures was evaluated online and offline according to the Racine's score: 1, mouth and facial movements; 2, head nodding; 3, forelimb clonus; 4, rearing; 5, rearing and falling (Racine, 1972). The number of wet-dog shakes in each stimulation trial was also counted, which negatively correlated with a fully kindled state (Lerner-Natoli et al., 1984).

\section{Optogenetics}

\section{Construction of optical cannulas}

One side of $0.39 \mathrm{NA}, \varnothing 200 \mu \mathrm{m}$ core multimode optical fibre (FT200EMT, Thorlabs) was terminated with a stainless-steel ferrule (SF230, Thorlabs). A fibre stripping tool was used to remove $10 \mathrm{~mm}$ of buffer (T12S21, Thorlabs). The TECS cladding was then removed using an acetone-soaked cotton bud. Following that, the exposed silica core was etched with hydrofluoric acid to form its pencil-like tip shape. This facilitated penetration and increased illumination volume. Current-output power relationships were examined on every optical cannula with a photodiode power sensor (S130C, Thorlabs) and a power meter (PM200, Thorlabs). The typical maximal power at the tip was set to $30-40 \mathrm{~mW}$.

\section{Construction of optoprobes}

A multimode $\varnothing 50 \mu \mathrm{m}$ optical fibre (FG050LGA, Thorlabs) was attached to each silicon probe based on a method previously described (Royer et al., 2010). Briefly, one side of the fibre was terminated with a stainless-steel LC ferrule (SFLC127, Thorlabs) in accordance with the manufacturer's guide (FN96A, Thorlabs). The acrylate coating of the other side was removed at a length of $1 \mathrm{~cm}$. Fluorine-doped cladding was then thinned with hydrofluoric acid while the silica core was kept intact. After that, each etched optical fibre was mounted on a single shank 32 channel silicon probe (A1x32-Poly2-10mm-50s-177H32_21mm; NeuroNexus) with a UV-curing optical adhesive (NOA61, Thorlabs). Current-output power relationships were examined on every optoprobe with a photodiode power sensor (S130C, Thorlabs) and a power metre (PM200, Thorlabs). The typical maximal power at the tip was set to $1-2 \mathrm{~mW}$.

\section{Light source and its control}

A $450 \mathrm{~nm}$ laser diode (PL450B; Osram) was driven via a current controller with analogue modulation (LDC205C; Thorlabs) and the emission beam was collimated with an aspheric lens. The collimated ray was divided and directed into two channel fibre ports, each of which was connected to a rat via multimode patch cables and a rotary joint. The laser power on each fibre port was controlled between $0 \mathrm{~mW}$ and $100 \mathrm{~mW}$ with a polarizing beam splitter, a half-wave plate, analogue modulation, and manual shutters (Supplementary Fig. 3). Laser power was stabilized with a self-made feedback control system consisting of a photo detector (PDA36A-EC, Thorlabs) and a microcomputer (STM32L152RE; STMicroelectronics). External command voltage was provided from a data acquisition board (USB-6212; National Instruments) or a pulse stimulator (Master-9; A.M.P.I.).

\section{Unit recordings of medial septum neurons and their} optogenetic modulation

Animals with ChR2 expression in the medial septum were anaesthetized with $1.5 \mathrm{~g} / \mathrm{kg}$ urethane (i.p.) and $0.1-0.3 \mathrm{mg} / \mathrm{kg}$ atropine (s.c.). They were then mounted on a stereotaxic instrument. Depth of anaesthesia was maintained by confirming the lack of nociceptive reflex. Each animal's rectal temperature was maintained at $36-37^{\circ} \mathrm{C}$ with a DC temperature controller. The head position was adjusted so that the bregma and lambda were at the same level. A small cranial window $(\sim 1 \mathrm{~mm}$ diameter) was made at $0.5 \mathrm{~mm}$ anterior from the bregma and $1 \mathrm{~mm}$ left of the midline. An optoprobe was inserted at a $9.5^{\circ}$ lefttilted angle from the parasagittal plane and gently advanced towards the medial septum. The electrode was kept and stabilized at several sites in the medial septum (5700-7000 $\mu \mathrm{m}$ from the pia) for the recordings. At each recording site, pulse and sine wave illuminations paired with non-illuminating epochs were applied to find optogenetically modulated units (Supplementary Fig. 4). The parameters for pulse illumination were as follows: duration: $10 \mathrm{~ms}$; amplitude: typically $1 \mathrm{~mW}$; frequency: 1,5 , 10, 20 and $40 \mathrm{~Hz}$; number of trials: 20; minimum number of cycles in each trial: 20; minimum trial duration: $5 \mathrm{~s}$. The parameters for sine wave illumination were as follows. Amplitude: typically $1 \mathrm{~mW}$; frequency: 1, 4, 8, 12 and $20 \mathrm{~Hz}$; number of trials: 20; minimum number of cycles at each trial: 10; minimum trial duration: 5 s. Illumination was conducted through a $450 \mathrm{~nm}$ laser diode (PL450B, Osram) driven via a current controller (LDC205C; Thorlabs) and a data acquisition board (USB-6212, National Instruments). DiD solution (2\% in ethanol, Thermo Fisher Scientific Cat\# D307) was applied to the non-recording side of the shank of optoprobes for the post hoc location of the recording sites.

\section{Seizure interventions via medial septum stimulation}

\section{Electrical and optogenetic stimulation}

During the chronic surgical implantation of the recording electrodes, either a bipolar stimulating electrode (the same construct as the kindling electrode) or an optical cannula targeting the medial septum was also implanted. The coordinates used were as follows: AP: $0.5 \mathrm{~mm}$ anterior from the bregma; ML: penetrated from $1.0 \mathrm{~mm}$ left of the midline at a $9^{\circ}$ left-tilted angle from the parasagittal plane; distance: $6.0 \mathrm{~mm}$ from the pia. The 
stimulating electrode or the optical cannula was fixed with dental cement. Medial septum stimuli for seizure interventions were commanded by TTL (transistor-transistor logic) signals either before (preconditioning) or after (closed-loop or open-loop) seizure induction by electrical stimulation (E-stim) of the HPC commissure. For E-stim of the medial septum, each TTL signal triggered a bipolar, $0.5 \mathrm{~ms}$ positive, $0.5 \mathrm{~ms}$ negative rectangular pulse at $\pm 400 \mu \mathrm{A}$ intensity. For optogenetic stimulation, each TTL signal triggered $30-\mathrm{ms}$ long square wave illumination at maximum intensity through each optical cannula (typically 30$40 \mathrm{~mW})$.

\section{Open-loop septal stimulation experiments}

Either electrical or optogenetic medial septum stimuli were delivered for $2 \mathrm{~min}$ at a fixed frequency $(1,8$ or $20 \mathrm{~Hz})$ following each seizure induction. These stimulus frequencies were chosen because low and theta-frequency stimulation of the limbic system during interictal periods alleviate TLE (Miller et al., 1994; Wang et al., 2016) and $20 \mathrm{~Hz}$, the maximum frequency medial septum stimulation, did not cause seizures in non-kindled rats in pilot experiments. The intensity of E-stim for seizure induction was determined either as the minimum intensity to reliably $(>90 \%)$ induce HPC after-discharges (suprathreshold stimulation: $\pm 10-100 \mu \mathrm{A}$ typically) or the maximum intensity at which HPC after-discharges were seldom $(<10 \%)$ induced (subthreshold stimulation: $\pm 5-50 \mu \mathrm{A}$ typically). Medial septum stimulated and non-stimulated trials were always performed in pairs, but their order was randomly chosen for each epoch (i.e. a set of a stimulated and a non-stimulated trial, e.g. on-off, off-on, off-on, on-off, etc). Both experiments were conducted using the same seizure-induction intensity within a given animal.

\section{Closed-loop septal stimulation experiments}

HPC electrographic seizure waves were detected online using a programmable digital signal processor unit (RX-8; TuckerDavis Technologies). These waves were used to trigger either electrical or optogenetic stimulation of the medial septum for on-demand real-time seizure interventions. Pre-amplified and multiplexed LFP signals were fed to the processor unit in parallel to the recording unit sampled at $500 \mathrm{~Hz}$. The signals were demultiplexed and analysed online to detect each LFP deflection in the HPC (mostly after-discharges) using a custom-made seizure detection algorithm based on a previously established routine (Kozák and Berényi, 2017). Briefly, LFP signals were demultiplexed at $500 \mathrm{~Hz}$ per channel and a signal from a preselected HPC channel was band-pass filtered with a fourth order Butterworth filter to $10-130 \mathrm{~Hz}$. Each single medial septum stimulus was triggered by an ictal HPC LFP deflection when a filtered signal exceeded a threshold. To avoid false positive detections of the stimulation artefacts, threshold crossings were allowed to trigger stimulation only if a preceding threshold crossing has happened within a pre-set time window (typically 30-300 ms) (Kozák and Berényi, 2017). Time resolution of the detection was $2 \mathrm{~ms}$. Both the threshold for amplitude and the duration of the time-window were fine-tuned for each animal. A delay $(0,20,40$ or $60 \mathrm{~ms})$ was introduced between detection and stimulation to target a specific phase of seizure waves (Fig. 1). The intensity of E-stim for seizure induction was determined as the minimum necessary for HPC after-discharges to be reliably $(>90 \%$ ) induced (suprathreshold stimulation: $\pm 10-100$ $\mu \mathrm{A}$ typically). Medial septum stimulated and non-stimulated trial pairs were always conducted with the same animals and with the same seizure induction intensity/stimulus delay. The on-off and off-on trial pairs were delivered in a pseudorandom order (e.g. on-off, off-on, off-on, on-off). Trials in which no after-discharges were observed were discarded.

\section{Preconditioning}

Either electrical or optogenetic medial septum stimuli were delivered for 5 or $60 \mathrm{~s}$ at $20 \mathrm{~Hz}$ before seizure induction. The intensity of seizure induction varied trial-by-trial to determine thresholds for HPC and Ctx electrographic seizures: regenerative HPC after-discharges and large amplitude oscillations in cortical LFPs associated with Racine's score 4 or 5 . The stimulus intensity typically started from $\pm 10 \mu \mathrm{A}$ and was incremented at \pm 10 $\mu \mathrm{A}$ steps. Medial septum stimulated and non-stimulated trials were always paired with a pseudorandom sequence of stimulus intensity.

\section{Immunohistochemistry}

\section{Colchicine injection}

To enhance the immunohistochemical reaction of cellular markers [glutamate and glutamate decarboxylase (GAD)], 150 $\mu \mathrm{g}$ of colchicine dissolved in saline (C9754; Sigma-Aldrich) was pressure-injected into the lateral ventricle $48 \mathrm{~h}$ before perfusion (Gritti et al., 2003). The stereotaxic coordinates of the injection site were: AP, $0.8 \mathrm{~mm}$ posterior from the bregma; ML, $1.5 \mathrm{~mm}$ right of the midline; DV, $4.4 \mathrm{~mm}$ from the pia.

\section{Perfusion and sectioning}

The animals were deeply anaesthetized with $1.5 \mathrm{~g} / \mathrm{kg}$ urethane (i.p.) and transcardially perfused with physiological saline followed by $4 \%$ paraformaldehyde (PFA) and $0.2 \%$ picric acid (PA) in 0.1 $\mathrm{M}$ phosphate buffer ( $\mathrm{pH} 7.2-7.3)$. After removal, brains were post-fixed in the same fixative overnight, embedded into $4 \%$ agarose, and sectioned in $40-50-\mu \mathrm{m}$ thick slices using a vibrating blade microtome (VT1000S, Leica). Sections were collected in 0.1 M PBS and then subjected to the staining described below. All staining procedures were performed at room temperature.

\section{NeuN immunohistochemistry}

All incubations were followed by washing with $0.3 \%$ Triton-X in $0.1 \mathrm{M}$ PBS (PBS-X). Sections were incubated successively with $10 \%$ normal goat serum (NGS) in PBS-X for $30 \mathrm{~min}$, 1:1000 diluted mouse anti-NeuN monoclonal antibody (Millipore Cat\# MAB377, RRID: AB_2298772) in PBS-X containing $1 \%$ normal donkey serum (NDS) and $0.02 \%$ sodium azide (PBS-XG) overnight, and $2 \mu \mathrm{g} / \mathrm{ml}$ Alexa Fluor ${ }^{(B)} 488$-conjugated goat anti-mouse IgG for $2 \mathrm{~h}$ (Thermo Fisher Scientific Cat\# A-11029, RRID: AB_2534088). Sections were then counterstained with fluoro-Nissl solution (Thermo Fisher Scientific). Sections were finally mounted on gelatine-coated glass slides, coverslipped with $50 \%$ glycerol and $2.5 \%$ triethylene diamine in PBS.

\section{GAD67/65 immunohistochemistry}

Somatic GAD expression levels were enhanced by colchicine, as described above. Sections were incubated successively with $10 \%$ NGS in PBS-X for $30 \mathrm{~min}, 1 \mu \mathrm{g} / \mathrm{ml}$ rabbit anti-GAD67/65 polyclonal antibody (Frontier Institute Cat\# GAD-Rb, RRID: AB_2571698) in PBS-XG overnight, and $2 \mu \mathrm{g} / \mathrm{ml}$ Alexa Fluor ${ }^{\circledR}$ 
A

\section{Generalization of seizures with HPC-origin}

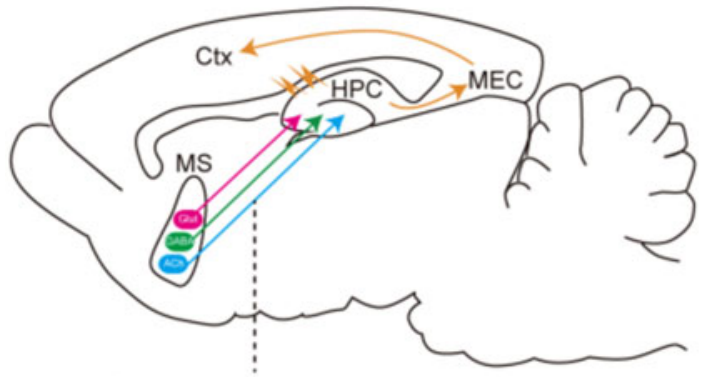

Septohippocampal pathway
B Seizure induction

MEC

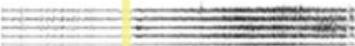

C

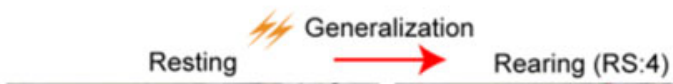

Ctx
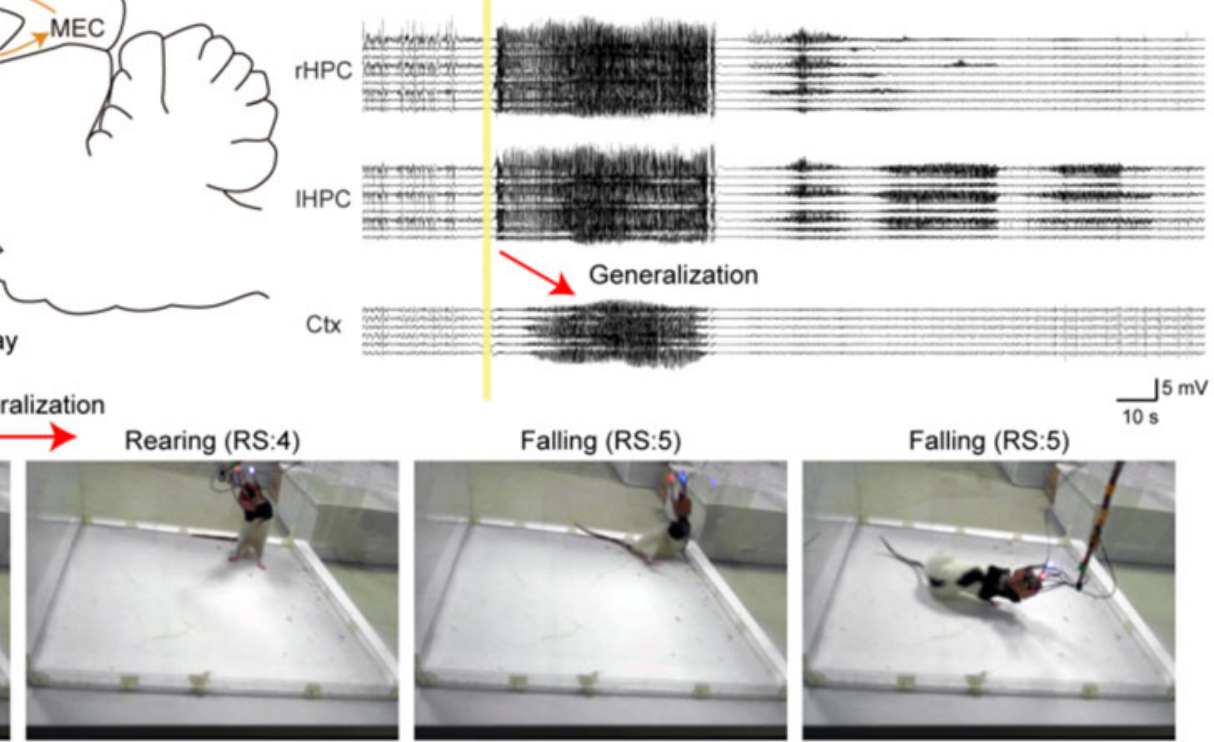

Falling (RS:5)

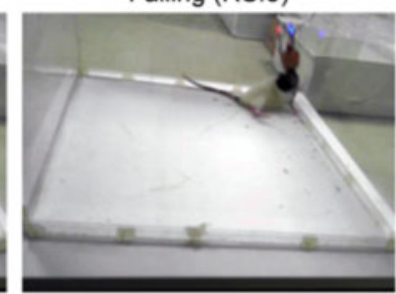

Falling (RS:5)
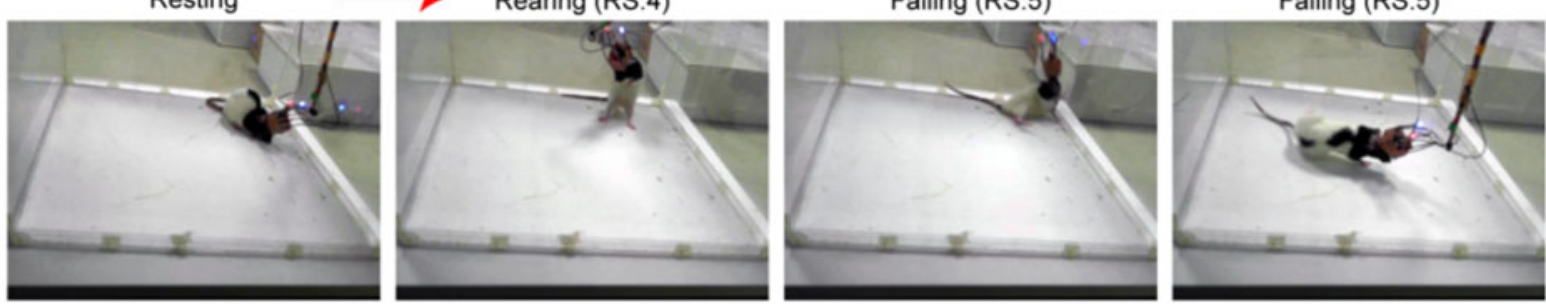

D(i)

Responsive open-loop intervention

On-line seizure detection

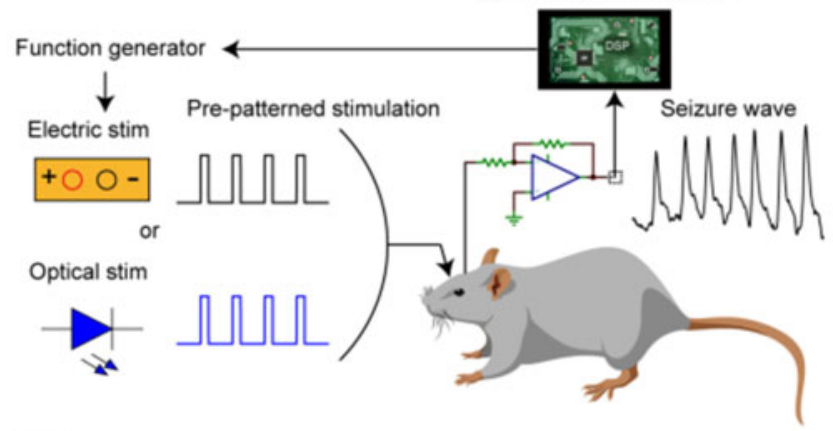

D(ii)

Responsive open-loop intervention Seizure induction

Seizure wave

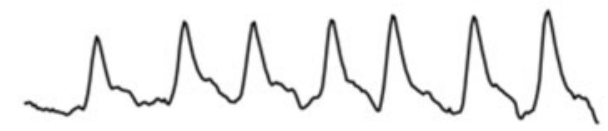

Stimulation

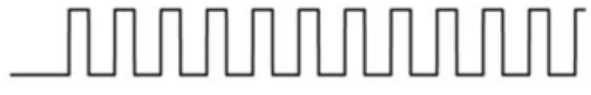

Stimulation at a fixed frequency and duration

\section{E(ii) Closed-loop seizure-rhythm intervention}

E(i) Closed-loop seizure-rhythm intervention

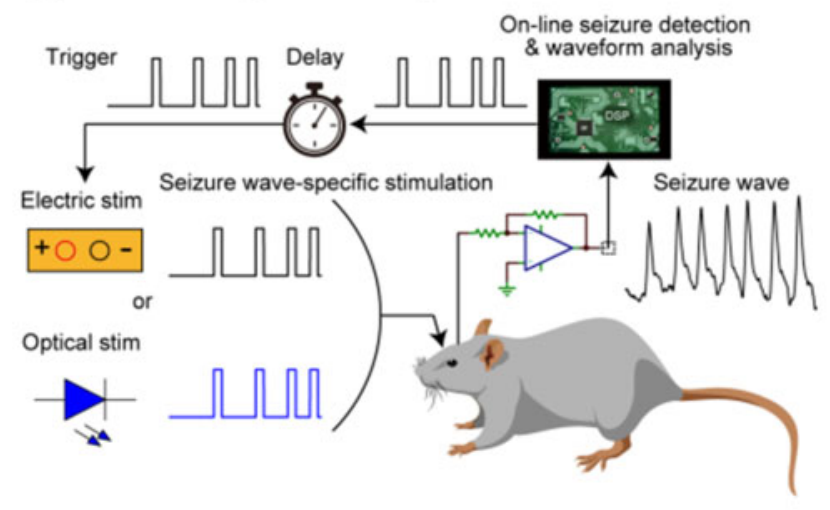

Figure I Responsive medial septum stimulation for intervening seizures of HPC-origin. (A) Schema of generalization of HPC seizures and septohippocampal pathway. MEC = medial entorhinal cortex. (B) Representative LFP traces of HPC seizures propagated to the Ctx. (C) E-stim of the HPC commissure also induced motor seizures. RS = Racine's score. [D(i) and (ii)] The concept of responsive open-loop intervention. Preprogrammed stimulation patterns (e.g. a pulse train of a fixed frequency and duration) were delivered after seizure induction. [E(i)] The concept of responsive closed-loop intervention (seizure rhythm-driven stimulation). Individual waves of provoked seizures were detected in real-time and each detection triggered medial septum stimulation with a defined delay. [E(ii)] A representative HPC seizure, its detection, and medial septum stimulation. Red arrows indicate detection and stimulation timings. See also Supplementary Figs I, 5 and 6 . MS = medial septum. 
A Open-loop MS electrical stimulation

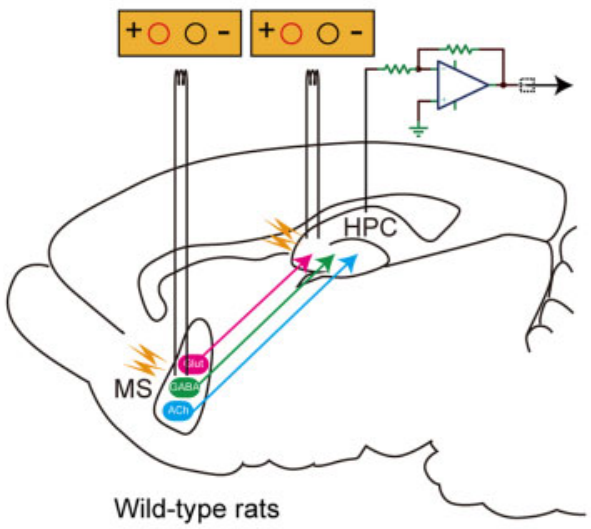

B

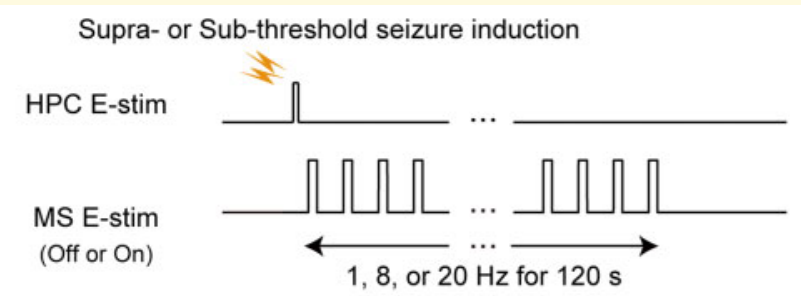

Supra-threshold seizure induction

C

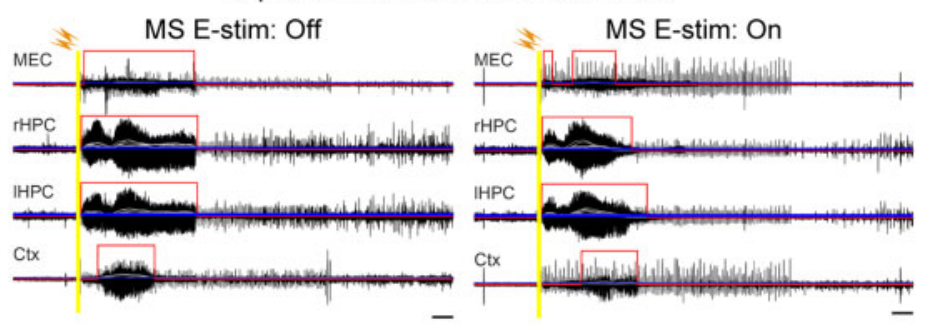

Supra-threshold seizure induction
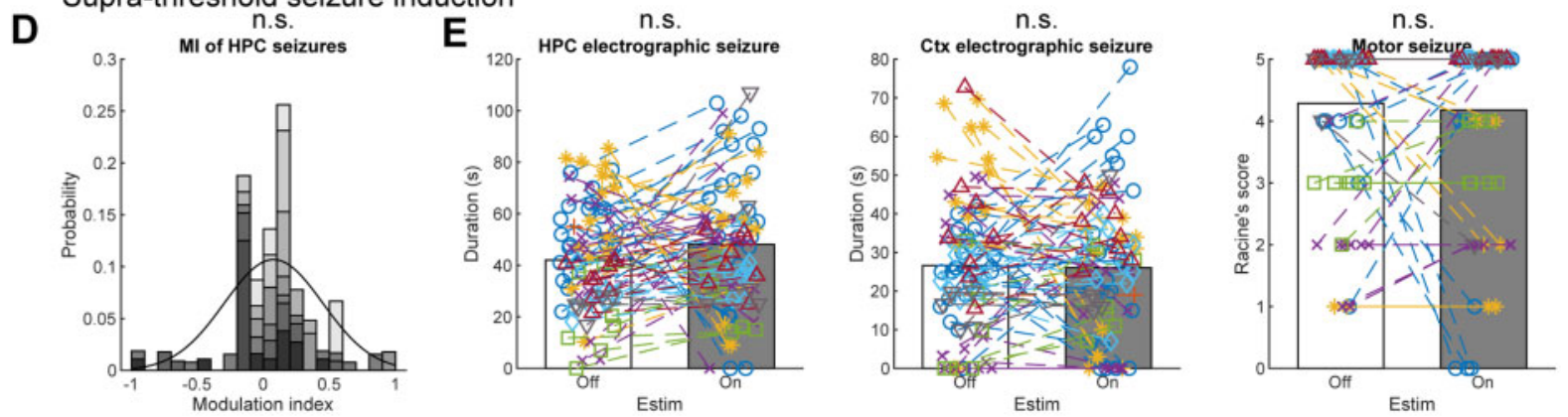

Sub-threshold seizure induction

$\mathbf{F}$

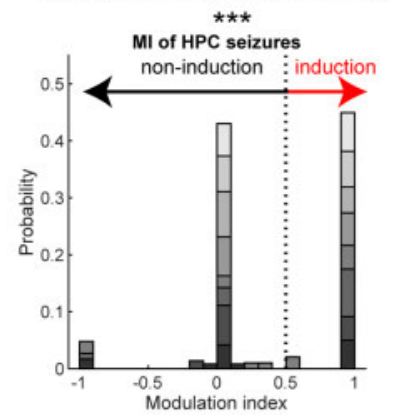

H

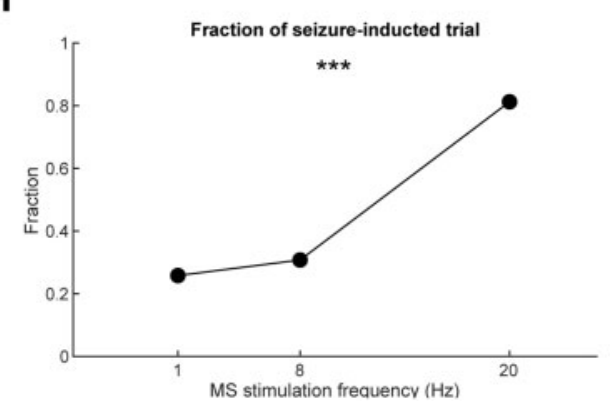

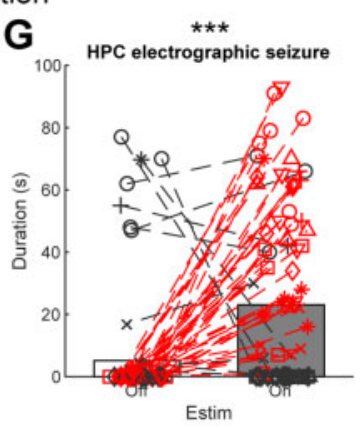

I

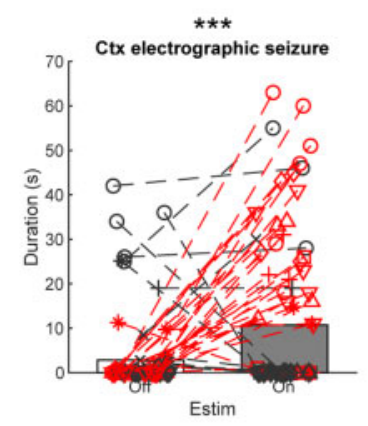

** E-stim on-off $x$ Frequency

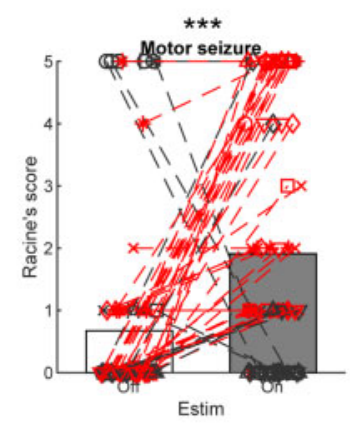

Estim

Figure 2 Open-loop medial septum E-stim has no effects or promotes HPC seizures. (A) Schema of the experiment. Stimulating electrodes were inserted into the HPC and the medial septum (MS) for seizure induction and intervention, respectively. (B) Open-loop responsive intervention. Seizures were induced at supra- or subthreshold intensity. (C) Representative seizure waves induced by the suprathreshold stimulation with or without open-loop medial septum E-stim. Six to nine 10-80 Hz bandpass-filtered LFP traces were overlapped for each brain region. Grey traces represent smoothed LFP traces with a moving average filter. Each blue line represents three times root-mean-square levels of the baseline activity for each smoothed LFP trace. Red lines represent detected seizures. Scale bars $=10 \mathrm{~s}$. (D and E) Results of open-loop intervention following suprathreshold commissural seizure induction. (D) Distribution of Mls of HPC seizure duration via medial septum E-stim. A black curve represents a fitted Gaussian model. (E) Durations of HPC and Ctx electrographic seizures, and motor seizure severity. Data of 
633-conjugated goat anti-rabbit IgG for $2 \mathrm{~h}$ (Thermo Fisher Scientific Cat\# A-21071, RRID: AB_2535732).

\section{Glutamate immunohistochemistry}

Somatic glutamate expression levels were enhanced by colchicine, as described above. The animals were perfused as described above except $0.5 \%$ glutaraldehyde was added to the fixative solution. Sections were incubated successively with $10 \%$ NDS in PBS-X for 30 min, 1:4000 diluted mouse anti-glutamate monoclonal antibody (ImmunoStar Cat\# 22523, RRID: AB_572244) in PBS-X containing $1 \%$ NDS and $0.02 \%$ sodium azide (PBS-XD) overnight, and $2 \mu \mathrm{g} / \mathrm{ml}$ Alexa Fluor ${ }^{\circledR} 647$-conjugated donkey anti-mouse IgG for $2 \mathrm{~h}$ (Thermo Fisher Scientific Cat\# A-31571, RRID: AB_162542).

\section{ChAT immunohistochemistry}

Sections were incubated successively with 10\% NDS in PBS-X for $30 \mathrm{~min}, 1: 500$ diluted goat anti-ChAT polyclonal antibody (Millipore Cat\# AB144P, RRID: AB_2079751) in PBS-XD overnight, and $2 \mu \mathrm{g} / \mathrm{ml}$ Alexa Fluor ${ }^{\circledR}$ 633-conjugated donkey antigoat IgG in PBS-XD for $2 \mathrm{~h}$ (Thermo Fisher Scientific Cat\# A21082, RRID: AB_2535739).

\section{cFos immunohistochemistry}

Just before perfusion, the medial septum with ChR2 transduction were laser-illuminated with $20 \mathrm{~Hz}$ sinusoidal waves $(20$ $\mathrm{mW}$ ) for $2 \mathrm{~h}$ in freely moving states, as described above. Sections were incubated successively with 10\% NGS in PBS-X for $30 \mathrm{~min}, 1: 4000$ diluted rabbit anti-cFos polyclonal antibody (Millipore Cat\# ABE457, RRID: AB_2631318) in PBS-XG overnight, and $4 \mu \mathrm{g} / \mathrm{ml}$ Alexa Fluor ${ }^{\circledR}$ 633-conjugated goat anti-rabbit IgG in PBS-XG for $2 \mathrm{~h}$ (Thermo Fisher Scientific).

\section{Concurrent GAD, glutamate and ChAT immunohistochemistry}

Somatic expression levels were enhanced by colchicine, as described above. Sections were incubated successively with $10 \%$ NDS in PBS-X for 30 min, a mixture of primary antibodies in PBS-XD overnight $(1 \mu \mathrm{g} / \mathrm{ml}$ rabbit anti-GAD67/65, Frontier; 1:4000 mouse anti-glutamate, ImmunoStar; 1:500 goat antiChAT, Millipore) and a mixture of secondary antibodies in PBX-XD for $2 \mathrm{~h}$ (donkey anti-goat, rabbit, and mouse IgGs conjugated with Alexa Fluor ${ }^{\circledR} 488,555$ and 647, respectively; $2 \mu \mathrm{g} /$ $\mathrm{ml}$ each; Thermo Fisher Scientific Cat\# A-11055, RRID: AB_2534102; Cat\# A-31572, RRID: AB_162543, and Cat\# A31571, RRID: AB_162542).

\section{GAD and PV double immunohistochemistry}

Somatic expression levels were enhanced by colchicine as described above. Sections were incubated successively with $10 \%$ NDS in PBS-X for $30 \mathrm{~min}$, a mixture of primary antibodies in PBS-XD overnight $(1 \mu \mathrm{g} / \mathrm{ml}$ rabbit anti-GAD67/65, Frontier and 1:3000 mouse anti-PV, Swant Cat\# 235, RRID: AB_10000343), and a mixture of secondary antibodies in PBS$\mathrm{XD}$ for $2 \mathrm{~h}$ (Alexa Fluor ${ }^{\circledR} 555$-conjugated donkey anti-rabbit IgG and Alexa Fluor ${ }^{\circledR}$ 647-conjugated donkey anti-mouse IgG; $2 \mu \mathrm{g} / \mathrm{ml}$, Thermo Fisher Scientific).

\section{Confocal microscopy}

Stained sections were examined using a Zeiss LSM880 scanning confocal microscope (Carl Zeiss). Images of 1-2 $\mu \mathrm{m}$ optical thickness were acquired using a Plan-Apochromat $40 \times / 1.4$ Oil DIC M27 or an alpha Plan-Apochromat $63 \times / 1.46$ Oil Korr M27 objective lens, $4.12 \mu$ s pixel time, and 16 times the frame average at $512 \times 512$ resolution. The illumination power ranged from $0.4-0.7 \mathrm{~mW}$, which did not induce obvious fading.

\section{Quantification and statistical analysis}

All data analyses were performed in MATLAB (RRID: SCR_001622; Mathworks, Natick, MA, USA) unless otherwise noted.

\section{Duration of electrographic seizures}

Wide-band $20 \mathrm{k}$ sample/s signals of open-loop intervention experiments were first downsampled to 1250 sample/s. They were then filtered with a third order zero phase lag Butterworth filter between 1 and $625 \mathrm{~Hz}$ to prepare LFP signals. Signals of closed-loop intervention experiments sampled at $500 \mathrm{~Hz}$ were filtered between 1 and $250 \mathrm{~Hz}$ for LFP signals. Peri-stimulus LFPs ( $30 \mathrm{~s}$ baseline and $180 \mathrm{~s}$ test epochs) were then extracted using timestamps recorded in a digital channel. The peri-stimulus LFP signals were further band-pass filtered to $10-80 \mathrm{~Hz}$ to prepare narrow-band LFPs. The narrow-band LFPs were then smoothed using a $3 \mathrm{~s}$ long moving average filter. The durations of the HPC and Ctx electrographic seizures were automatically detected and defined in test epochs when all amplitudes of the smoothed LFPs in each brain region exceeded three times the root-mean-square levels of the corresponding baseline epochs. The durations of electrographic seizures with medial septum

Figure 2 Continued

different medial septum stimulus frequencies were pooled. Each marker pair with a dashed line represents a pair of consecutive non-medial septum-stimulated and medial septum-stimulated trials with the same HPC commissure stimulus intensity and the same delay in the same rat. Values are represented as bars for means and circles for each trial. (F-I) Results of open-loop intervention following subthreshold commissural seizure induction. (F) Distribution of Mls. Each trial pair was clustered into non-seizure induction or seizure induction group (see 'Materials and methods' section). (G) The same conventions as in $\mathbf{E}$. Red coloured data represent the seizure-induction cluster. (H) Fractions of seizure-induced trials against medial septum E-stim frequencies. (I) Medial septum E-stim frequency resolved representation of the Ctx seizure durations and motor seizure scores. Data from each rat were represented as a dedicated monochrome tone in $\mathbf{D}$ and $\mathbf{F}$, and a dedicated colour and/or marker type in E and G. Values are represented as means \pm SD, $n=168$ and 178 trials from eight rats for $\mathbf{D}$ and $\mathbf{E}$, and $\mathbf{F}-\mathbf{I}$, respectively. Statistical significance was tested by two-sample Kolmogorov-Smirnov for asymmetry (skewness) of Ml distribution (D and F), paired $t$-test for seizure durations and Wilcoxon singed-rank test for the Racine's score $(\mathbf{E}$ and $\mathbf{G}), \chi^{2}$ test $(\mathbf{H})$, and three-way repeated ANOVA (I). Results of statistical tests are extensively reported in Supplementary Table I. See also Supplementary Fig. I4. MEC = medial entorhinal cortex; n.s. = not significant; RS = Racine's score. ${ }^{* *} P<0.01$, ***P $<0.001$. 
electrical interventions were refined by the consensus estimate of two experienced researchers doing manual inspections and using Neuroscope software (RRID: SCR_002455) (Hazan et al., 2006). This refinement was carried out because the automated detection algorithm sometimes misestimated durations due to the electrical artefacts. The coefficient of variation between the manual estimates of both researchers and the automated detection data of optogenetic interventions was $<5 \%$.

\section{Modulation index of hippocampus electrographic seizures}

The modulation index (MI) of the duration of HPC electrographic seizures via medial septum stimulation was defined as (HPC seizure duration with medial septum stimulation - HPC seizure duration without medial septum stimulation) / (HPC seizure duration with medial septum stimulation + HPC seizure duration without medial septum stimulation). The MI distributions were fitted with a Gaussian mixture model for clustering of possible subpopulations with anti-seizure effects ('Success' and 'Non-success' trials) or pro-seizure effects ('Induction' or 'Non-induction' trials). A threshold was determined for each experimental dataset as being the MI value at which the two Gaussian curves cross. However, for Fig. 2F, a threshold MI of 0.5 based on other datasets was used because that distribution could not be well fitted to a Gaussian mixture model. The skewness (asymmetry) of MI distributions was evaluated by comparing the absolute value distributions of positive and negative MIs.

\section{Assessment of the severity of motor seizures}

The severity of motor seizures was video monitored and evaluated according to Racine's scale on each trial (Racine, 1972).

\section{Temporal distribution and phase- locking strength of medial septal stimulations}

Signals were filtered between 1 and $250 \mathrm{~Hz}$ and downsampled (500 sample/s) to obtain LFP signals. The LFP signals in the HPC were further band-pass filtered to $10-25 \mathrm{~Hz}$ to prepare narrow-band LFPs. The instantaneous phase of the narrowband signal was calculated via Hilbert transform. The detection and/or stimulation timestamps of HPC LFP deflections driving the medial septum intervention were recorded at $20 \mathrm{k}$ or 500 $\mathrm{Hz}$ via the analogue channels of the recording device, in synchrony with the neuronal data. HPC LFP phase values corresponding to the real-time detection and/or stimulation timestamps were extracted, and the resultant vectors of the phase distributions of the corresponding to the detection and/or stimulation timings per seizure were calculated using Circular Statistics Toolbox (Berens, 2009). The phase-locking strength $(r$ = length of the resultant vector) and target angle (theta) were calculated for each trial. The distribution of $r$ was fitted by a two component Gaussian mixture model. A threshold was then determined where the two Gaussian curves cross. Based on the threshold, closed-loop medial septum E-stim trials were classified into low $r$ and high $r$ groups for stimulus phase-locking resolved analyses.

\section{Power spectral analysis of pre-ictal local field potential with respect to the intervention outcome}

Signals $30 \mathrm{~s}$ preceding each seizure induction were extracted, down-sampled to $500 \mathrm{~Hz}$ and $<250 \mathrm{~Hz}$ low-pass filtered on each recording site to obtain LFP signals. The LFP signals were then labelled by the outcome of the closed-loop medial septum E-stim intervention (i.e. success or non-success). The power spectra of the LFP signals were calculated using Chronux Toolbox across a 1-s sliding window with a $50 \%$ overlap (Bokil et al., 2010). The powers of the LFP signals in distinct frequency bands $(1-4 \mathrm{~Hz}, 4-12 \mathrm{~Hz}, 13-30 \mathrm{~Hz}, 30-45 \mathrm{~Hz}, 45-90 \mathrm{~Hz})$ were compared between the success and non-success groups.

\section{Spike sorting and unit classification}

Neuronal spikes were detected from the digitally high-pass filtered raw signal $(0.5-5 \mathrm{kHz})$ by a threshold crossing-based algorithm. Detected spikes were automatically sorted using a masked EM algorithm for Gaussian mixtures (Kadir et al., 2014) implemented in Klusta software, which is an open-source, automatic spike sorting package (https://github.com/kwikteam/ klusta) (Supplementary Fig. 4). This automatic clustering process was followed by the manual refinement of the clusters using KlustaViewa software to obtain well-isolated single units (Rossant et al., 2016) (https:/github.com/klusta-team/klusta viewa/). Multi-unit and noise clusters were discarded during this manual process. The quality of cluster isolation was estimated by calculating the isolation distance and interspike interval index for each cluster as previously described (Berényi et al., 2014); poor quality clusters were discarded.

\section{Detection of optogenetically modulated units}

For the detection of modulated units by $10 \mathrm{~ms}$ pulse illumination, peri-stimulus time histograms (PSTH) were prepared and cross-correlation (CCG) analysis was conducted between stimulus and spike timestamps (Supplementary Fig. 4). Significant modulation of the in-firing rate of each unit was identified using a shuffling method. For each stimulus and unit pair, surrogate datasets of timestamps were constructed by the random permutation of labels: either stimulus or unit 200 times. Pointwise $95 \%$ acceptance bands were calculated from the surrogate dataset CCGs for each $1 \mathrm{~ms}$ bin. Multiple comparison error was corrected by introducing 'global significance bands' (Fujisawa et al., 2008). The 10-ms illumination was considered to have excitatory or inhibitory effects on the referred unit if any of its CCG bins reached above or below these global bands within the considered time window.

For the detection of modulated units by sinusoidal illumination, instantaneous phase values of stimulus waves were calculated via Hilbert transform and the significant distribution of each unit on the phase was tested using the Rayleigh test in CircStat MATLAB Toolbox with a 0.05 alpha level (Berens, 2009). 


\section{Detection of modulated units by septal local field potentials}

LFP signals were calculated on each recording site in the medial septum by $1250 \mathrm{~Hz}$ down-sampling and $<300 \mathrm{~Hz}$ low-pass filtering. The LFP signals were averaged (Supplementary Fig. 4). The averaged LFP signal was then band-pass filtered into five frequency bands (delta, 1-4 Hz; theta, 4-12 Hz; beta, 12-30 $\mathrm{Hz}$; low-gamma, 30-45 Hz; mid-gamma, 45-80 Hz; highgamma, 80-150 Hz) with a third order zero phase-lag Butterworth filter. Instantaneous phase values were calculated using Hilbert transform. Significant modulations of each unit by these LFP frequency bands were tested using the Rayleigh test.

\section{Image analysis}

Acquired raw image files were opened and ChR2-mCherry and immunohistochemical signals were automatically and linearly enhanced with Carl Zeiss software (ZEN Digital Imaging for Light Microscopy, RRID: SCR_013672). Each image was exported as an 8-bit tiff file. Co-localizations between ChR2mCherry signals and the immunohistochemical reactions of celltype markers were manually inspected with the Cell Counter plugin of ImageJ software (RRID: SCR_003070, NIH, Bethesda, MD, USA).

\section{Statistical analysis}

Values are given as mean \pm standard deviation (SD) unless otherwise noted. MATLAB with Statistics and Machine Learning Toolbox was used for the statistical tests. Two-tailed paired $t$-test was used to compare the means of electrographic seizure durations and the threshold intensity of seizure induction with or without septal preconditioning. Wilcoxon singed-rank test was used to compare Racine's score values between trials with or without medial septum stimulation. The $\chi^{2}$ test was used to examine biased fractions of MI-based clustering (seizure induction trials or success trials) over a medial septum stimulus condition (frequency or delay). Three-way repeated measure ANOVA was used to examine consistent interactions between medial septum stimulation and clustering based on the MI of seizure parameters (HPC seizure duration, Ctx seizure duration, and Racine's score). Spearman's rank correlation test was used to examine correlations between measures [e.g. the MI of HPC seizure duration versus changes in Racine's score via medial septum stimulation, the phase-locking strength $(r)$ versus Racine's score]. Two-sample Kolmogorov-Smirnov test was used to compare the absolute value distributions of positive and negative MIs and the distributions of HPC seizure durations with or without medial septum stimulation to evaluate skewness. Oneway ANOVA followed by Bonferroni correction was used to test the effect of medial septum E-stim or illumination, delay, the seizure period and the types of intervention on the phaselocking strength of medial septum stimulation over HPC seizure waves. An unpaired $t$-test was used to compare the power spectra difference between success and non-success trials and to compare phase-locking strength between 0-20 s and 20-60 s after seizure onsets. The 10th lower percentile and the 90th upper percentile were used to remove outliers in each group. The significance level was set at $P<0.05$. One, two and three asterisks denote significance levels $<0.05,<0.01$ and $<0.001$, respectively. Data were analysed and the results are presented both across animals and per animal where applicable. Scientists performing the behavioural scoring of the motor seizures were blind to the stimulation conditions. No other blinding techniques could be used because of the obvious stimulation visibility in the recordings. All statistics are fully reported in Supplementary Table 1.

\section{Data availability}

All of the raw data, codes of figure generation and codes for data analysis are available online (Supplementary Table 2).

\section{Results}

\section{Responsive medial septum stimulation for intervening seizures of hippocampal origin}

HPC-kindled rats were used as a model of refractory TLE (Gelinas et al., 2016); rats were fully kindled by daily E-stim of their HPC commissure $(n=29)$. In the kindled rats, the commissure stimulation instantaneously induced massive $10-25 \mathrm{~Hz}$ after-discharges in their bilateral HPCs (Fig. 1A and $\mathrm{B})$. These HPC seizures were then secondary generalized, resulting in Racine's score 4 or 5 motor seizures (Fig. 1A, C and Supplementary Fig. 2). To terminate these seizures, we focused on two types of responsive medial septum E-stim; we call these open-loop and closed-loop interventions (Fig. 1D and E). For open-loop intervention, stimulus trains at fixed rates were delivered following seizure induction (i.e. in response to seizure episodes). For closedloop intervention, stimulus pulses were triggered by the individual ictal LFP deflection in the HPC, meaning that the medial septum was stimulated with the internal ongoing rhythmicity of HPC seizures for their entire duration (closed-loop seizure-rhythm stimulation) (Supplementary Figs 5 and 6). A delay of 0 to $60 \mathrm{~ms}$ was introduced to shift the stimulus pulses to target specific phases of the seizure waves (Kozák and Berényi, 2017).

\section{Open-loop medial septum electrical stimulation has no effect or promotes hippocampal seizures}

Open-loop responsive medial septum E-stim was examined first (Fig. 2A). Each medial septum E-stim $( \pm 400 \mu \mathrm{A}$ biphasic, $1 \mathrm{~ms}$-long) evoked robust and transient potentials in the HPC and the EC $(1-4 \mathrm{mV})$, but not in the Ctx (Supplementary Fig. 1A and C). Following the seizure induction, medial septum E-stim trains were delivered at 1,8 or $20 \mathrm{~Hz}$ for $2 \mathrm{~min}$ (Fig. 2B); those stimuli themselves did not cause any seizures in non-kindled rats (data not shown). The open-loop medial septum E-stim did not shorten HPC electrographic seizures at any frequencies examined $(42.0 \pm 20.5 \mathrm{~s}$ to $48.1 \pm 24.5 \mathrm{~s}$ per trial, $P>0.05$, paired $t$-test; $38.9 \pm 15.0$ s to $44.7 \pm 9.9$ s per animal, $P>0.05$; Fig. $2 \mathrm{C}, \mathrm{E}$ 
A Closed-loop MS electrical stimulation

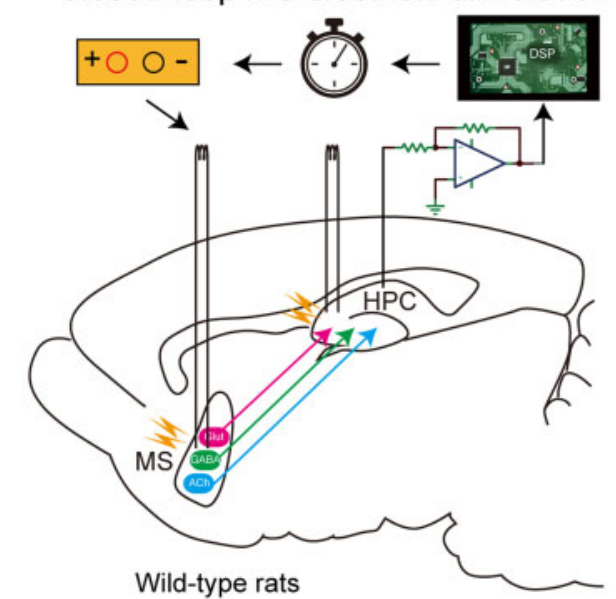

D
B

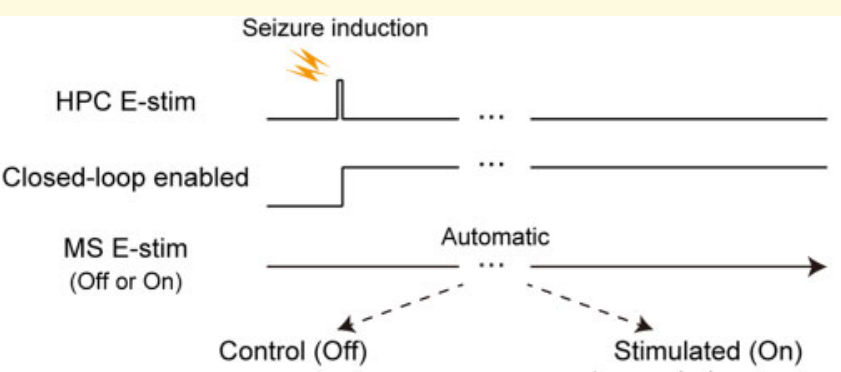

C

HPC LFP

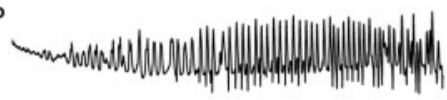

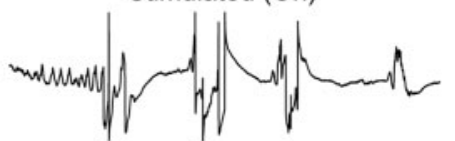

Detection

MS E-stim
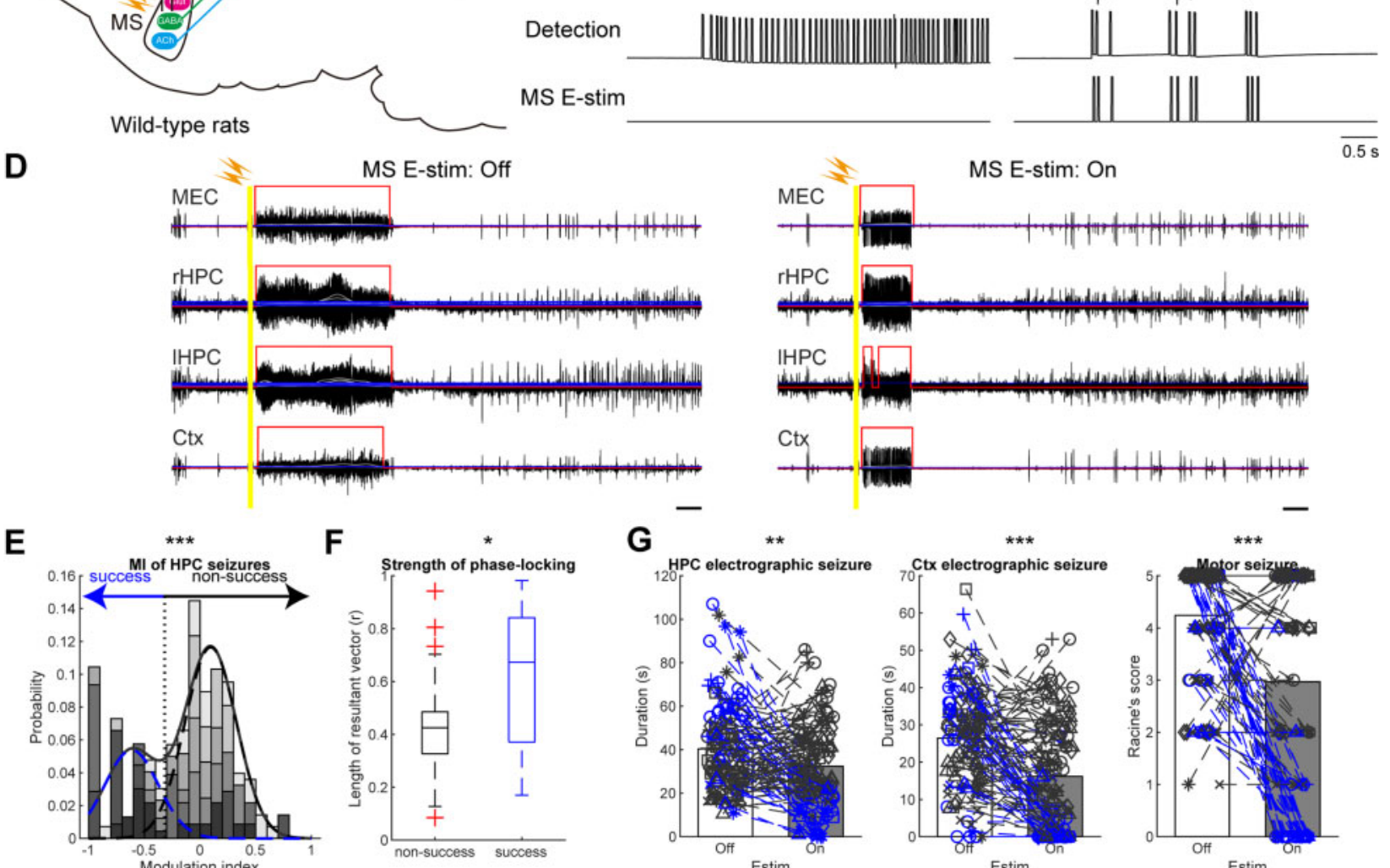

H

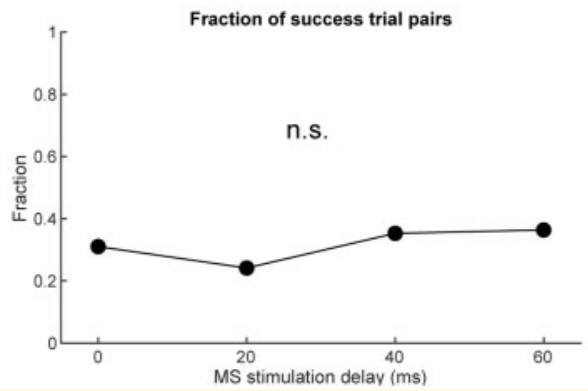

G

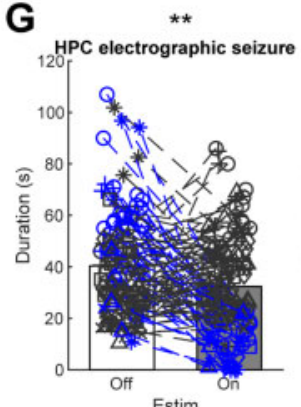

I

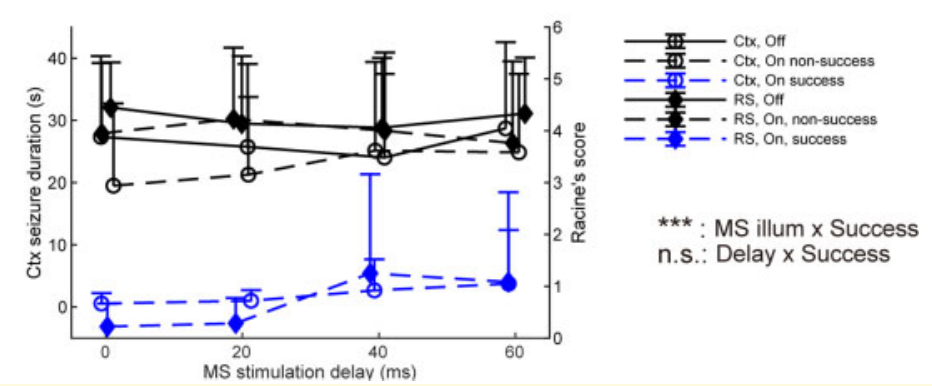

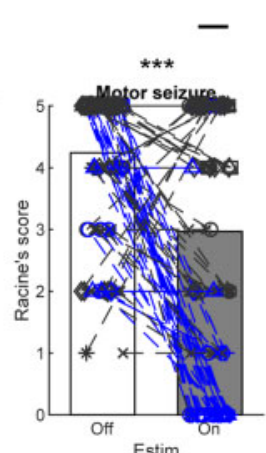
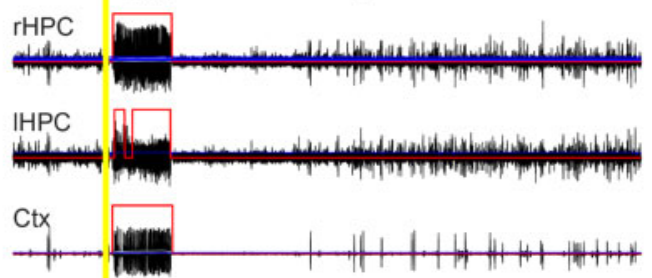

Figure 3 Closed-loop seizure rhythm-driven medial septum E-stim terminates seizures of HPC-origin. (A) Schema of the experiment. (B) Close-loop seizure rhythm intervention. Seizures were induced by suprathreshold commissural stimulation. Automatic seizure detection was turned on immediately after seizure induction. Each detection of HPC LFP deflection triggered a medial septum (MS) E-stim pulse with a preset delay. (C) HPC seizure waves with or without the medial septum E-stim. (D) Representative seizure waves with or without the medial septum E-stim. The same convention is used as in Fig. 2C. (E) Distribution of Mls. The distribution was fitted to a Gaussian mixture model and each trial pair was clustered into success or non-success groups using a threshold where the two Gaussians crossed. (F) Phase-locking strengths $(r)$ of medial septum E-stim on HPC seizure waves of success and non-success trials during $20 \mathrm{~s}$ following seizure onsets. Data from trials using 0 ms stimulus delay are presented. (G) Durations of HPC and Ctx electrographic seizures, and motor seizure severity with the clusters defined in E: blue, success trial pairs; black, non-success trial pairs. Data with different delays were pooled. $(\mathbf{H})$ Fractions of success trial pairs as a function of delays. (I) Delay time resolved representation of the Ctx seizure durations and motor seizures scores. $n=250$ trials from seven rats. Other conventions for data presentation and statistical tests carried out are the same as in Fig. 2. See also Supplementary Figs 5,12 and I5. $* P<0.05$, $* * P<0.01$, *** $P<0.001$. 
and Supplementary Fig. 7). Trial numbers, animal numbers, and other statistics are fully reported (figure legends and Supplementary Table 1). MIs of the HPC seizures by medial septum E-stim were distributed around 0 and this distribution was not skewed, which indicated almost no modulation $(P>0.05$, two-sample Kolmogorov-Smirnov test; Fig. 2D). Neither Ctx electrographic nor motor seizures were alleviated by the stimulation $(28.3 \pm 19.4 \mathrm{~s}$ to $25.2 \pm 20.0 \mathrm{~s}$ and $4.2 \pm 1.4$ to $4.1 \pm 1.6$ in Racine's score per trial, $P>0.05$ for both, paired $t$-test and Wilcoxon singed-rank test, respectively; $26.9 \pm 11.6 \mathrm{~s}$ to $23.1 \pm 5.8 \mathrm{~s}$ and $4.3 \pm 0.7$ to $4.2 \pm 0.5$ per animal, $P>0.05$ for both; Fig. 2C, E and Supplementary Fig. 7).

We then examined the effects of this open-loop medial septum E-stim on incomplete seizure episodes evoked by subthreshold commissure stimulation, which typically resulted in non-generalized partial seizures (Fig. 2F-I). The open-loop medial septum E-stim significantly elongated HPC seizures $(5.2 \pm 16.9 \mathrm{~s}$ to $23.1 \pm 28.0 \mathrm{~s}$ per trial, $P<0.001$, paired $t$-test; $4.4 \pm 6.9 \mathrm{~s}$ to $22.7 \pm 10.3 \mathrm{~s}$ per animal, $P<0.001$; Fig. $2 \mathrm{G}$ and Supplementary Fig. 8). To underpin a factor for this pro-seizure effect, stimulated and non-stimulated trial pairs were classified into seizure-induction $(\mathrm{MI}>0.5)$ and non-seizure-induction trials $(\mathrm{MI} \leqslant 0.5)$ $(P<0.001$, two-sample Kolmogorov-Smirnov test; Fig. $2 \mathrm{~F})$. The fraction of seizure-induction trials was significantly higher at $20 \mathrm{~Hz}(0.813)$ than those at $1 \mathrm{~Hz}$ and $8 \mathrm{~Hz}(0.258$ and 0.308 , respectively; $P<0.001, \chi^{2}$ test; Fig. $\left.2 \mathrm{H}\right)$. The open-loop medial septum E-stim significantly deteriorated Ctx and motor seizures $(2.8 \pm 8.3 \mathrm{~s}$ to $10.7 \pm 8.3 \mathrm{~s}$ and $0.7 \pm 1.5$ to $1.9 \pm 2.1$ in Racine's score per trial, $P<0.001$ for both, paired $t$-test and Wilcoxon signed-rank test; $2.4 \pm 3.7 \mathrm{~s}$ to $10.2 \pm 8.2 \mathrm{~s}$ and $0.6 \pm 0.7$ to $1.9 \pm 0.7$ per animal, $P<0.05$ for both; Fig. $2 \mathrm{G}$ and Supplementary Fig. 8). Notably, $20 \mathrm{~Hz}$ stimulation induced longer Ctx seizures $(121.2 \pm 19.4 \mathrm{~s})$ and higher Racine's score values $(3.7 \pm 1.6)$ in seizure-induction trials (Fig. 2I). It is worthwhile noting that open-loop medial septum E-stim at higher frequency $(\geqslant 40 \mathrm{~Hz})$ induced generalized episodes of motor seizures with Racine scale 4 or 5 even in non-kindled rats (data not shown). These results suggest that open-loop medial septum E-stim does not alleviate HPC seizures and that a false positive triggering of an open-loop responsive stimulator can even make seizures worse (Supplementary Fig. 9).

\section{Closed-loop seizure rhythm-driven medial septum electrical stimulation terminates seizures of hippocampal origin}

Medial septum E-stim was then delivered in a closed-loop manner (Fig. 3A and B). The induced HPC seizures were processed with a custom-made algorithm in real-time and each HPC LFP deflection in the $10-130 \mathrm{~Hz}$ range triggered a single-pulse medial septum E-stim $( \pm 400 \mu \mathrm{A}$ biphasic, 1$\mathrm{ms}$ long) with a fixed delay. In contrast to the open-loop stimulation, closed-loop seizure rhythm-driven medial septum E-stim significantly shortened HPC seizures compared to those in the interwoven, non-stimulated trials $(40.3 \pm 20.3$ $\mathrm{s}$ to $32.4 \pm 20.0 \mathrm{~s}$ per trial, $P<0.01$, paired $t$-test; $40.2 \pm 14.4$ s to $30.6 \pm 6.5$ s per animal, $P=0.179$; Fig. $3 \mathrm{C}$, D, G and Supplementary Fig. 10). The closed-loop stimulation also shortened Ctx seizures $(26.4 \pm 13.4 \mathrm{~s}$ to $16.2 \pm 14.8 \mathrm{~s}$ per trial, $P<0.001$, paired $t$-test; $27.5 \pm 7.9 \mathrm{~s}$ to $15.3 \pm 6.6 \mathrm{~s}$ per animal, $P<0.05)$ and decreased seizure severity according to Racine's scale $(4.2 \pm 1.2$ to $3.0 \pm 2.1$ per trial, $P<0.001$, Wilcoxon signed-rank test; $4.3 \pm 0.7$ to $2.9 \pm 1.2$ per animal, $P<0.05$ ). Because these seizure terminating effects were in an all-or-none manner, the MI distribution of HPC seizures could be fitted with a twocomponent Gaussian mixture model and off-on trial pairs were classified into success and non-success trials $(P<0.001$, two-sample Kolmogorov-Smirnov test; Fig. 3E). The success and non-success trials had distinct LFP power spectra in the S1, M1, bilateral HPCs, and MEC during the $30 \mathrm{~s}$ just before seizure inductions (Supplementary Fig. 11). However, their spectral difference was not confined to a specific frequency range. Fractions of success trials were not significantly different between delays $(0.310,0.241,0.353$ and 0.364 for $0,20,40$ and $60 \mathrm{~ms}$, respectively; $P>0.05$, $\chi^{2}$ test; Fig. $\left.3 \mathrm{H}\right)$. The success and non-success classifications were also consistently valid for the Ctx and motor seizures; interaction between medial septum E-stim and success labelling were significant for Ctx and motor seizures $(P<0.001$ for both, three-way repeated ANOVA; Fig. 3I). The outcomes were consistent for all stimulus delays $(P>0.05)$. On success trials, the average duration of Ctx seizures and Racine's score values were robustly reduced $(<3.7 \mathrm{~s}$ and $<1.3 \mathrm{~s}$; Fig. $3 \mathrm{H}$ ), which meant secondary generalization was effectively suppressed. These results indicate strong seizure terminating effects of the closed-loop medial septum E-stim (Supplementary Fig. 9).

It is noteworthy that for the first $20 \mathrm{~s}$ of the seizures the phase-locking strengths of medial septum E-stim on HPC seizure rhythms were significantly higher in success trials than those in non-success trials $(0.522 \pm 0.231$ and $0.423 \pm 0.234$, respectively; $P<0.05$, unpaired $t$-test, Fig. $3 \mathrm{~F}$ and Supplementary Fig. 12A). The distribution of the phaselocking strengths ( $r$ values) of medial septum E-stim on HPC seizure rhythms seemed bimodal (Supplementary Fig. 12B). Therefore, we fitted their distribution with a two component Gaussian mixture model to determine a threshold. The closed-loop medial septum E-stim trials were then classified into 'low $r$ ' and 'high $r$ ' groups, where the stimulation patterns were loosely and strongly coupled to the ictal HPC LFP rhythms, respectively. The durations of cortical electrographic seizures and the severity of the motor seizures in the strongly coupled group were significantly smaller than those in loosely coupled group $(10.4 \pm 11.3 \mathrm{~s}$ and $17.0 \pm 15.0 \mathrm{~s}$, $2.2 \pm 2.0$ and $3.1 \pm 2.1 \mathrm{~s}$ per trial, $P<0.05$, unpaired $t$-test; $9.6 \pm 9.9 \mathrm{~s}$ and $15.3 \pm 6.4 \mathrm{~s}, 2.5 \pm 1.6$ and $2.9 \pm 1.2 \mathrm{~s}$ per animal; Supplementary Fig. 12C). The durations of HPC electrographic seizures in the 'high $r$ ' group tended to be smaller 
than those in 'low $r$ ' group $(26.2 \pm 11.9 \mathrm{~s}$ and $33.0 \pm 21.3 \mathrm{~s}$ per trial, $P=0.104$, unpaired $t$-test; $27.4 \pm 3.6 \mathrm{~s}$ and $31.3 \pm 7.1 \mathrm{~s}$ per animal; Supplementary Fig. 12C). The durations of HPC and Ctx electrographic seizures and Racine's value all negatively correlated with the stimulus coupling (correlation coefficient rho: $-0.318,-0.262$ and -0.320 , respectively, $* * P<0.001$, Spearman's rank correlation test, Supplementary Fig. 12D). These correlations indicate that medial septum E-stim resulted in successful outcomes when the intervention was well phase-locked on the HPC seizure rhythms.

\section{Seizure-terminating effects of closed-loop stimulation are mediated by medial septum}

\section{neurons}

Medial septum E-stim might activate not only medial septum neurons but also medial septum targeting HPC neurons via antidromic spikes, nearby brain regions by current spread, and even glial cells (Monai et al., 2016; Tsanov, 2018). To investigate if the seizure terminating effects are mediated specifically by medial septum neurons, we used optogenetic stimulation (Supplementary Figs 3 and 4). ChR2 was transduced to medial septum neurons using a viral vector with a synapsin promoter (Supplementary Figs 13-15). The gene transduction was confined to the medial septum (Supplementary Fig. 1B) and ChR2 signals were well colocalized with neuronal marker NeuN immunoreactions (Supplementary Figs 13 and 14B). Medial septum blue-light illumination induced cFos expression in ChR2-expressing neurons and the modulated unit activities of medial septum neurons. This resulted in the successful activation of medial septum neurons (Supplementary Figs 13 and 14C). Each medial septum illumination reliably induced robust and fast LFP deflections in the HPC and the EC but not in the Ctx (Supplementary Fig. 1D). Similar to E-stim, open-loop medial septum illumination did not alleviate HPC seizures $(43.6 \pm 22.4 \mathrm{~s}$ to $40.8 \pm 18.9 \mathrm{~s}$ per trial, $P>0.05$, paired $t$ test; $43.6 \pm 11.3 \mathrm{~s}$ to $40.3 \pm 6.3 \mathrm{~s}$ per animal, $P>0.05$; Supplementary Fig. 14A, D, E and G). The MI distribution was not skewed $(P>0.05$, two-sample KolmogorovSmirnov test; Supplementary Fig. 14F). Neither Ctx nor motor seizures were affected by the open-loop illumination $(32.1 \pm 14.1 \mathrm{~s}$ to $30.9 \pm 14.5 \mathrm{~s}$ and $4.5 \pm 0.9 \mathrm{~s}$ to $4.3 \pm 1.2 \mathrm{~s}$ in Racine's score per trial, $P>0.05$ for both, $t$-test and Wilcoxon signed rank test, respectively; $32.0 \pm 4.8 \mathrm{~s}$ to $30.6 \pm 3.1 \mathrm{~s}$ and $4.4 \pm 0.6 \mathrm{~s}$ to $4.3 \pm 0.7 \mathrm{~s}$ per animal, $P>0.05$ for both; Supplementary Fig. 14G). These results further indicate that open-loop medial septum stimulation is not effective in terminating seizures of HPC-origin.

The closed-loop seizure rhythm-driven activation of nonspecific medial septum neurons was then examined in the same rats (Supplementary Fig. 15A and B). In contrast to open-loop illumination, closed-loop seizure rhythm medial septum illumination significantly shortened HPC seizures compared to those in the interwoven, non-illuminated trials $(48.2 \pm 16.6 \mathrm{~s}$ to $33.0 \pm 25.5 \mathrm{~s}$ per trial, $P<0.001$, paired $t$ test; $42.5 \pm 12.6 \mathrm{~s}$ to $30.8 \pm 14.4 \mathrm{~s}$ per animal, $P=0.146$; Supplementary Fig. 15C and E). The illumination also shortened Ctx seizures and decreased Racine's score values compared to those in the non-illuminated trials $(34.9 \pm 20.0 \mathrm{~s}$ to $23.0 \pm 20.2 \mathrm{~s}$ and $4.2 \pm 1.3 \mathrm{~s}$ to $3.0 \pm 2.0 \mathrm{~s}$ per trial, $P<0.001$ for both, paired $t$-test and the Wilcoxon signedrank test; $26.7 \pm 17.4 \mathrm{~s}$ to $18.3 \pm 12.8 \mathrm{~s}$ and $3.7 \pm 1.5 \mathrm{~s}$ to $2.6 \pm 1.2 \mathrm{~s}$ per animal, $P=0.184$ and 0.125 , respectively; Supplementary Fig. 15C and E). The MI distribution was well fitted to a two-component Gaussian mixture model; one of the Gaussians was centred around -1 and the other was around zero $(P<0.01$, two-sample KolmogorovSmirnov test; Supplementary Fig. 15D). Illuminated and non-illuminated trial pairs were then classified into success and non-success trial pairs based on the model. This classification was consistent with all seizure parameters examined $(P<0.001$, three-way ANOVA; Supplementary Fig. 15G). Seizure manifestations were significantly alleviated in success trials $(<3.9 \mathrm{~s},<5.4 \mathrm{~s}$ and $<1.9$ in Racine's score for HPC seizures, Ctx seizures, and motor seizures, respectively; Supplementary Fig. 15G). Fractions of success trials were not significantly different between delays $(0.471,0.444$, 0.125 and 0.267 for $0,20,40$ and 60 ms delays, respectively; $P>0.05, \chi^{2}$ test; Supplementary Fig. $\left.15 \mathrm{~F}\right)$. Thus, closedloop seizure rhythm activation of medial septum neurons has seizure terminating effects compatible to those of medial septum E-stim. These results suggest that the seizure-terminating effects of closed-loop seizure rhythm-driven medial septum E-stim are mediated by medial septum neurons.

\section{Seizure-terminating effects of closed-loop medial septum stimulation are mediated by GABAergic medial septum population}

We next dissected the contributions of the GABAergic, glutamatergic and cholinergic medial septum neurons to the observed seizure-terminating effects (Supplementary Fig. 16). Cell-type specific Cre-dependent expression of virally transduced ChR2 was established in three transgenic lines. As the VGAT- and CaMKII $\alpha$-Cre driver lines were newly developed using CRISPR-Cas9 technology, we confirmed the cell-type specific ChR2 expression, and their proper electrophysiological functionality (Supplementary Figs 3, 4 and 17-19).

Closed-loop activation of septal GABAergic neurons prevents generalization of hippocampal seizures

The medial septum of VGAT-Cre driver rats were injected with a Cre-dependent ChR2-expressing viral vector (Fig. 4A). ChR2 signals were predominantly co-localized with GAD65/67 immunoreactions, but not with those of choline acetyltransferase (ChAT) (Fig. 4B and Supplementary Fig. 17). Some ChR2 signals were co- 
A

Closed-loop MS GABAergic activation

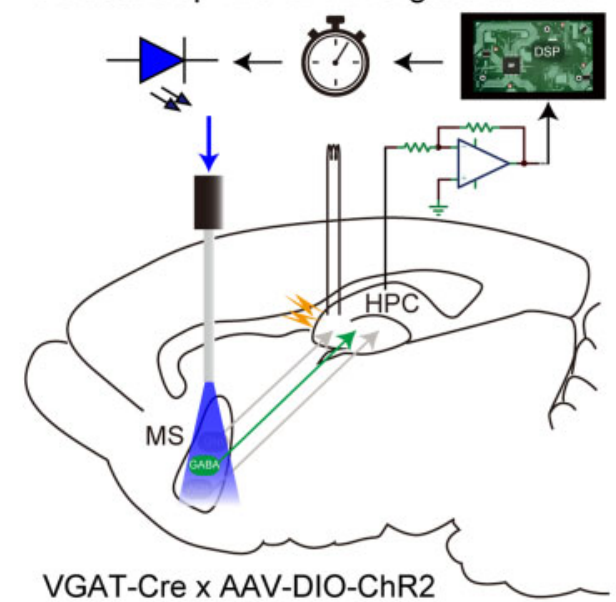

B

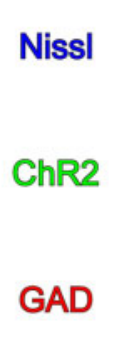

D

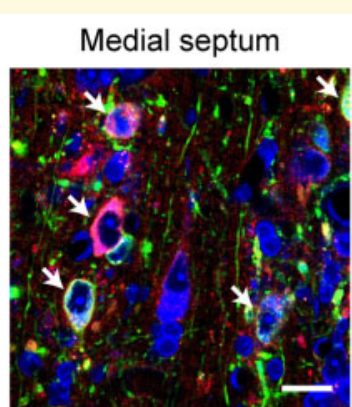

C

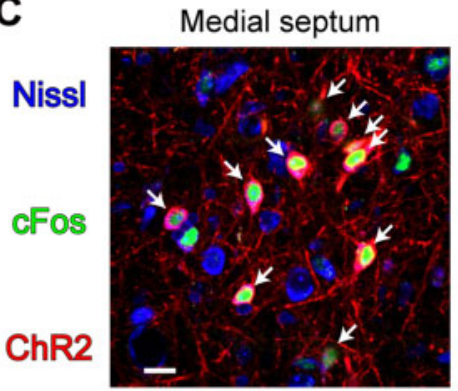

Seizure induction

HPC E-stim

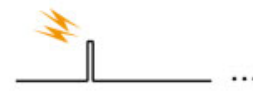

Closed-loop enabled
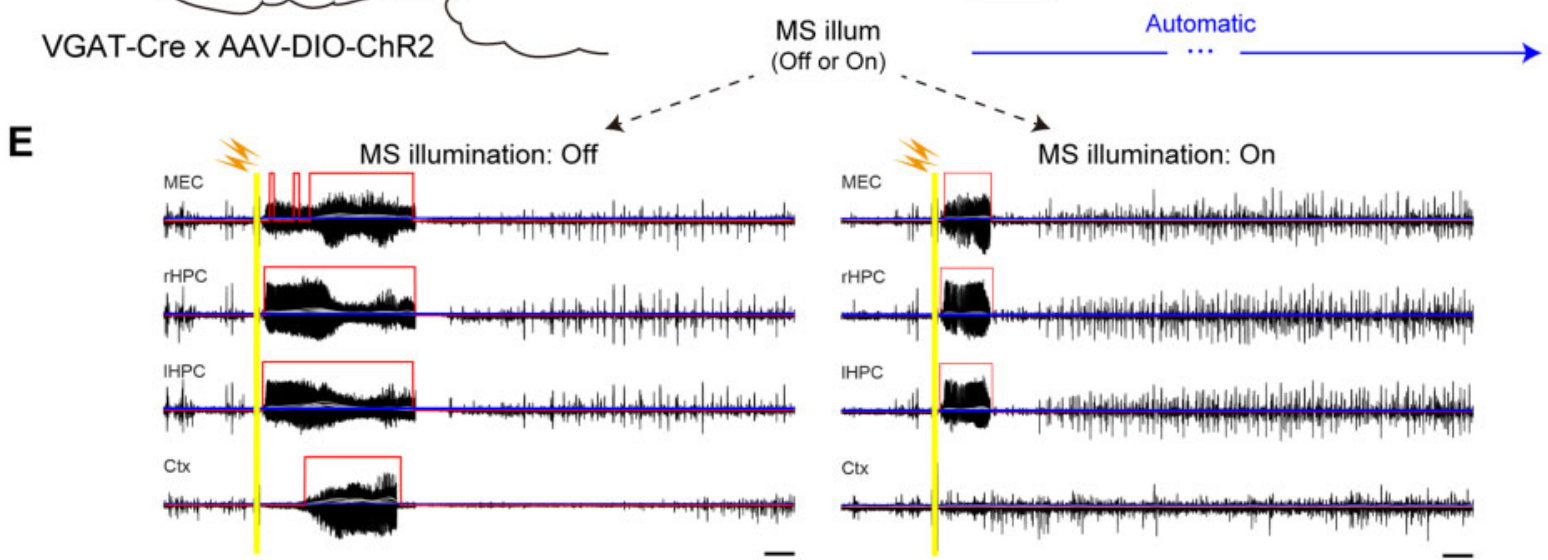

$\mathbf{F}$ ****

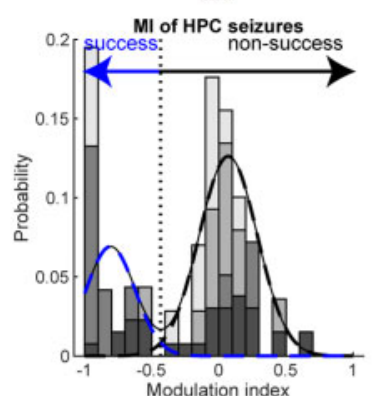

G

H

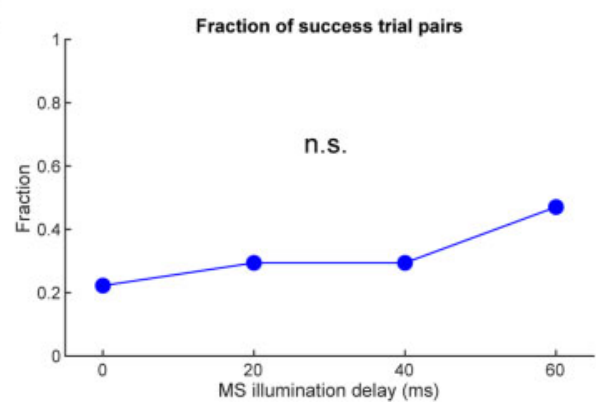

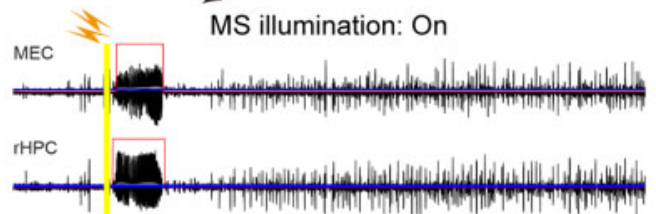

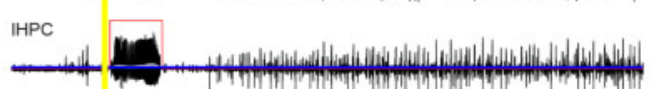

CDx

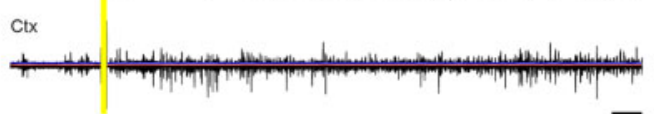

$\star \star \star *$

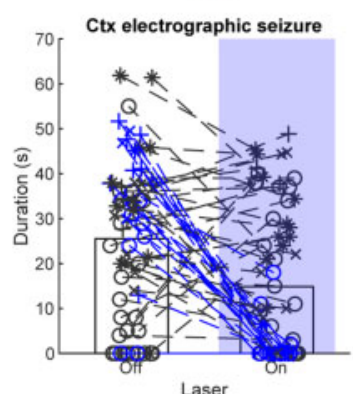

Laser

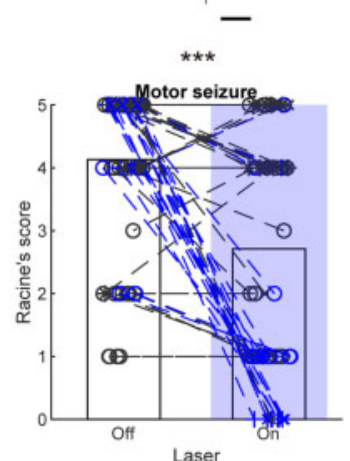

I

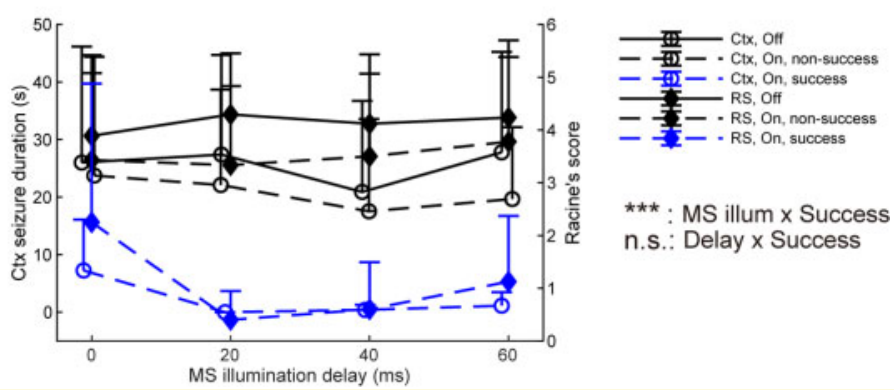

Figure 4 Closed-loop seizure rhythm-driven activation of medial septum GABAergic neurons terminates seizures of HPC origin. (A) Schema of the experiment. (B) ChR2 signals were co-localized with GABAergic neurons. Arrows indicate co-localizations. Scale bar $=20 \mu \mathrm{m}$. (C) cFos expression was co-localized with ChR2 expressing neurons after illumination. Arrows indicates co-localizations. Scale bar $=20 \mu \mathrm{m}$. (D) Closed-loop seizure rhythm intervention. Each detection triggered a 30 ms-long medial septum (MS) illumination with a fixed delay. (E) Representative seizures with or without the medial septum illumination. (F) Distribution of seizure Mls with and without medial septum illumination. The distribution was fitted with a Gaussian mixture model and each trial pair was clustered into success or non-success trial groups. (G) Durations of HPC and Ctx electrographic seizures, and motor seizure severity with the clusters defined in F. (H) Fractions of success trial pairs as a function of delays. (I) Medial septum illumination delay resolved representation of the $\mathrm{Ctx}$ seizure durations and motor seizure scores. $n=138$ trials from four rats. Other conventions are the same as in Fig. 3. See also Supplementary Fig. 21 . n.s. = not significant; RS $=$ Racine's score. ${ }^{* * P}<<0.01$, **** $<<0.001$. 
localized with glutamate reactions because glutamate is a precursor of GABA, and medial septum GABAergic neurons use glutamate as local transmitters (Gritti et al., 2003). Medial septum illumination of the rats induced cFos expression in ChR2-expressing neurons (Fig. 4C) and LFP deflections in the HPC and the EC, but not in the Ctx (Supplementary Fig. 1E). Similar to medial septum E-stim and hSyn::ChR2 illumination, the closed-loop seizure rhythm activation of medial septum GABAergic neurons significantly shortened HPC seizures compared to those in the interwoven, non-illuminated trials $(47.4 \pm 21.9 \mathrm{~s}$ to $34.6 \pm 18.3 \mathrm{~s}$ per trial, $P<0.01$, paired $t$-test; $50.4 \pm 6.8 \mathrm{~s}$ to $34.9 \pm 17.3$ s per animal, $P=0.165$; Fig. $4 D, E, G$ and Supplementary Fig. 20). The closed-loop GABAergic activation also shortened Ctx seizures and decreased Racine's score values compared to the non-illuminated trials $(25.5 \pm 7.6 \mathrm{~s}$ to $14.8 \pm 16.6 \mathrm{~s}$ and $4.1 \pm 1.3 \mathrm{~s}$ to $2.7 \pm 1.9 \mathrm{~s}$ per trial, $P<0.001$ for both, paired $t$-test and Wilcoxon signedrank test, respectively; $29.9 \pm 10.2 \mathrm{~s}$ to $16.0 \pm 7.9 \mathrm{~s}$ and $4.4 \pm 0.7 \mathrm{~s}$ to $2.8 \pm 0.9 \mathrm{~s}$ per animal, $P=0.073$ and 0.125 , respectively; Fig. 4E, G and Supplementary Fig. 20). Illumination and non-illumination trial pairs were classified into success and non-success trial pairs as before $(P<0.001$, two-sample Kolmogorov-Smirnov test; Fig. 4F). This classification was consistently valid on both Ctx and motor seizures $(P<0.001$ for all, three-way ANOVA; Fig. 4G and I). Ctx seizures on success trials were quite short and Racine's scores were $<3$, which indicated that secondary generalization was effectively suppressed $(<7.25 \mathrm{~s}$ and $<2.25$ in Racine's score; Fig. 4I). The fractions of success trials were not significantly different over delays $\left(P>0.05, \chi^{2}\right.$ test; Fig. $4 \mathrm{H})$. These results suggest that GABAergic neurons mediate the seizure-terminating effects of closed-loop medial septum stimulation.

The open-loop activation of medial septum GABAergic neurons was also investigated (Supplementary Fig. 21A and B). However, open-loop medial septum GABAergic activation did not shorten HPC or Ctx seizures and did not decrease Racine's score values $(49.6 \pm 21.8 \mathrm{~s}$ to $45.5 \pm 27.4 \mathrm{~s}$, $27.0 \pm 16.1 \mathrm{~s}$ to $24.1 \pm 16.4 \mathrm{~s}$, and $4.3 \pm 1.0 \mathrm{~s}$ to $3.9 \pm 1.2 \mathrm{~s}$ per trial, $P>0.05$ for all; $51.3 \pm 10.0 \mathrm{~s}$ to $44.1 \pm 10.2 \mathrm{~s}$, $29.0 \pm 9.0 \mathrm{~s}$ to $24.4 \pm 9.2 \mathrm{~s}$, and $4.5 \pm 0.7 \mathrm{~s}$ to $4.0 \pm 0.4 \mathrm{~s}$ per animal, $P>0.05$; Supplementary Fig. 21C-E). These results suggest that seizure rhythm congruent activation is also crucial for the seizure-terminating effects of medial septum GABAergic neurons.

\section{Neither closed-loop nor open-loop activation of medial septum glutamatergic neurons alleviates seizures of hippocampal origin}

The roles of medial septum glutamatergic neuronal populations were then investigated (Supplementary Fig. 22). The medial septum of CaMKII $\alpha$-Cre driver rats were injected with a Cre-dependent ChR2-expressing viral vector (Supplementary Fig. 18A); ChR2 signals were predominantly co-localized with glutamate immunoreactions, but not with those of GAD67 or ChAT. Medial septum illumination in the rats induced cFos expression in ChR2-expressing neurons (Supplementary Fig. 18B). It also induced fast LFP deflections in the HPC and the EC but not in the Ctx (Supplementary Fig. 1E). The closed-loop seizure rhythm activation of medial septum glutamatergic neurons did not decrease the duration of HPC and Ctx seizures and Racine's scores in the population data $(53.2 \pm 20.9 \mathrm{~s}$ to $50.6 \pm 17.2 \mathrm{~s}$, $27.8 \pm 15.4 \mathrm{~s}$ to $26.4 \pm 15.0 \mathrm{~s}$, and $4.5 \pm 1.1 \mathrm{~s}$ to $4.2 \pm 1.5 \mathrm{~s}$ per trial, $P>0.05$ for all; $51.5 \pm 9.4 \mathrm{~s}$ to $49.6 \pm 7.3 \mathrm{~s}$, $27.1 \pm 4.8 \mathrm{~s}$ to $25.5 \pm 4.9 \mathrm{~s}$, and $4.5 \pm 0.4 \mathrm{~s}$ to $4.1 \pm 0.7 \mathrm{~s}$ per animal, $P>0.05$ for all; Supplementary Fig. $22 \mathrm{C}$ and E). The MIs of HPC seizures had a mode around zero (Supplementary Fig. 22D).

Open-loop medial septum glutamatergic activation was also investigated. However, it did not shorten HPC or Ctx seizures and did not decrease Racine's scores at all $(48.4 \pm 13.4 \mathrm{~s}$ to $55.2 \pm 17.1 \mathrm{~s}, 39.0 \pm 13.9 \mathrm{~s}$ to $38.8 \pm 15.5 \mathrm{~s}$, and $4.6 \pm 1.0 \mathrm{~s}$ to $4.3 \pm 1.3 \mathrm{~s}$ per trial, $P=0.01,0.91$ and 0.10 , respectively; $48.0 \pm 7.2 \mathrm{~s}$ to $54.7 \pm 8.9 \mathrm{~s}, 38.8 \pm 7.6 \mathrm{~s}$ to $38.5 \pm 10.4 \mathrm{~s}$, and $4.6 \pm 0.6 \mathrm{~s}$ to $4.3 \pm 0.5 \mathrm{~s}$ per animal, $P=0.008,0.904$ and 0.250 , respectively; Supplementary Fig. 22H-J).

Together, these results suggest that the activation of the medial septum glutamatergic neuronal population alone does not effectively terminate HPC origin seizures.

\section{Neither closed-loop nor open-loop activation of medial septum cholinergic neurons alleviates seizures of hippocampal origin}

The medial septum of ChAT-Cre driver rats were injected with a Cre-dependent ChR2-expressing viral vector (Fig. 5D and Supplementary Fig. 23A and F). ChR2 signals were predominantly co-localized with ChAT immunoreactions, but not with those of GAD65/67 (Fig. 5B and Supplementary Fig. 19). Some ChR2 signals were co-localized with glutamate reactions as well, presumably because glutamate is also a local transmitter in medial septum cholinergic neurons (Gritti et al., 2003). Medial septum illumination in the rats did not induce the deflection of HPC LFPs (Supplementary Fig. 1E), but did induced cFos expression in ChR2-expressing neurons (Fig. 5C and Supplementary Fig. 19). In contrast to GABAergic and glutamatergic activation, the closed-loop seizure-rhythm activation of medial septum cholinergic neurons did not alleviate HPC, Ctx or motor seizures $(55.0 \pm 17.8$ s to $51.2 \pm 21.4 \mathrm{~s}, 39.2 \pm 17.8 \mathrm{~s}$ to $36.0 \pm 19.5 \mathrm{~s}$, and $4.8 \pm 0.6 \mathrm{~s}$ to $4.8 \pm 0.8 \mathrm{~s}$ per trial, $P>0.05$ for all; $51.5 \pm 9.4 \mathrm{~s}$ to $49.6 \pm 7.3 \mathrm{~s}, 27.1 \pm 4.8 \mathrm{~s}$ to $25.5 \pm 4.9 \mathrm{~s}$, and $4.4 \pm 0.4 \mathrm{~s}$ to $4.1 \pm 0.7 \mathrm{~s}$ per animal, $P>0.05$ for all; Supplementary Fig. 23B, C and E). The MI distribution was not skewed $(P>0.05$, two-sample Kolmogorov-Smirnov test, Supplementary Fig. 23D).

Open-loop activation of the medial septum cholinergic neurons was then examined but it again did not shorten HPC, Ctx or motor seizures $(51.8 \pm 30.7 \mathrm{~s}$ to $53.5 \pm 29.6 \mathrm{~s}$, $29.6 \pm 20.6 \mathrm{~s}$ to $32.5 \pm 22.5 \mathrm{~s}$, and $4.1 \pm 1.6 \mathrm{~s}$ to $3.9 \pm 1.6 \mathrm{~s}$ per trial, $P>0.05 ; 48.0 \pm 7.2 \mathrm{~s}$ to $54.7 \pm 8.9 \mathrm{~s}, 38.8 \pm 7.6 \mathrm{~s}$ to $38.5 \pm 10.4 \mathrm{~s}$, and $4.6 \pm 0.6 \mathrm{~s}$ to $4.3 \pm 0.5 \mathrm{~s}$ per animal, 
A

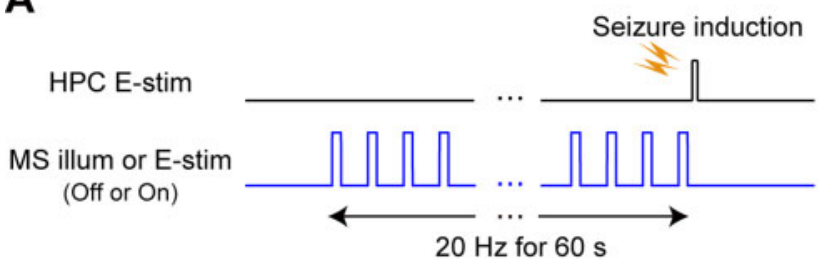

D MS cholinergic pre-conditioning

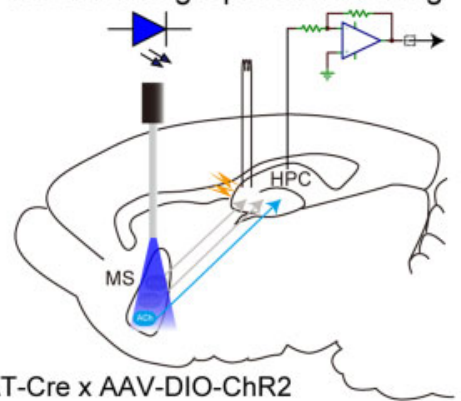

$\mathbf{F}$ ChAT-Cre x AAV-DIO-ChR2
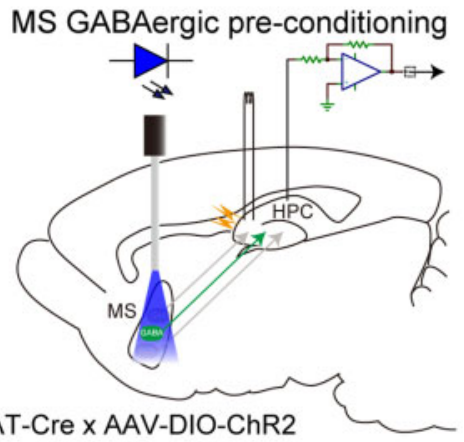

H MS glutamatergic pre-conditioning

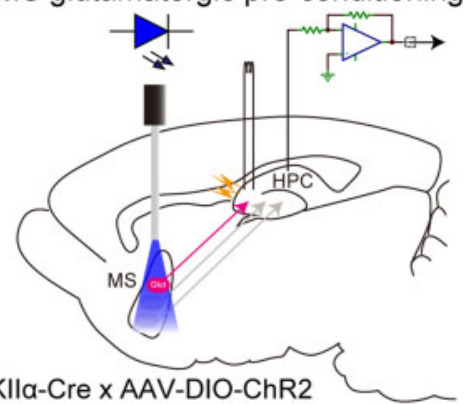

CaMKIla-Cre x AAV-DIO-ChR2

J

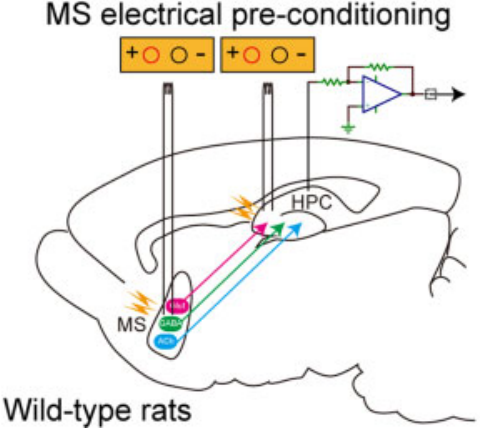

E

G

I

K

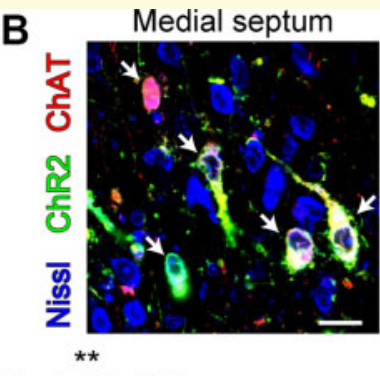

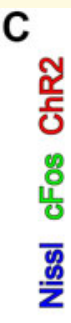

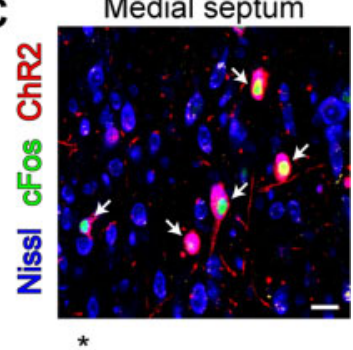

Thresshold for ctox 0
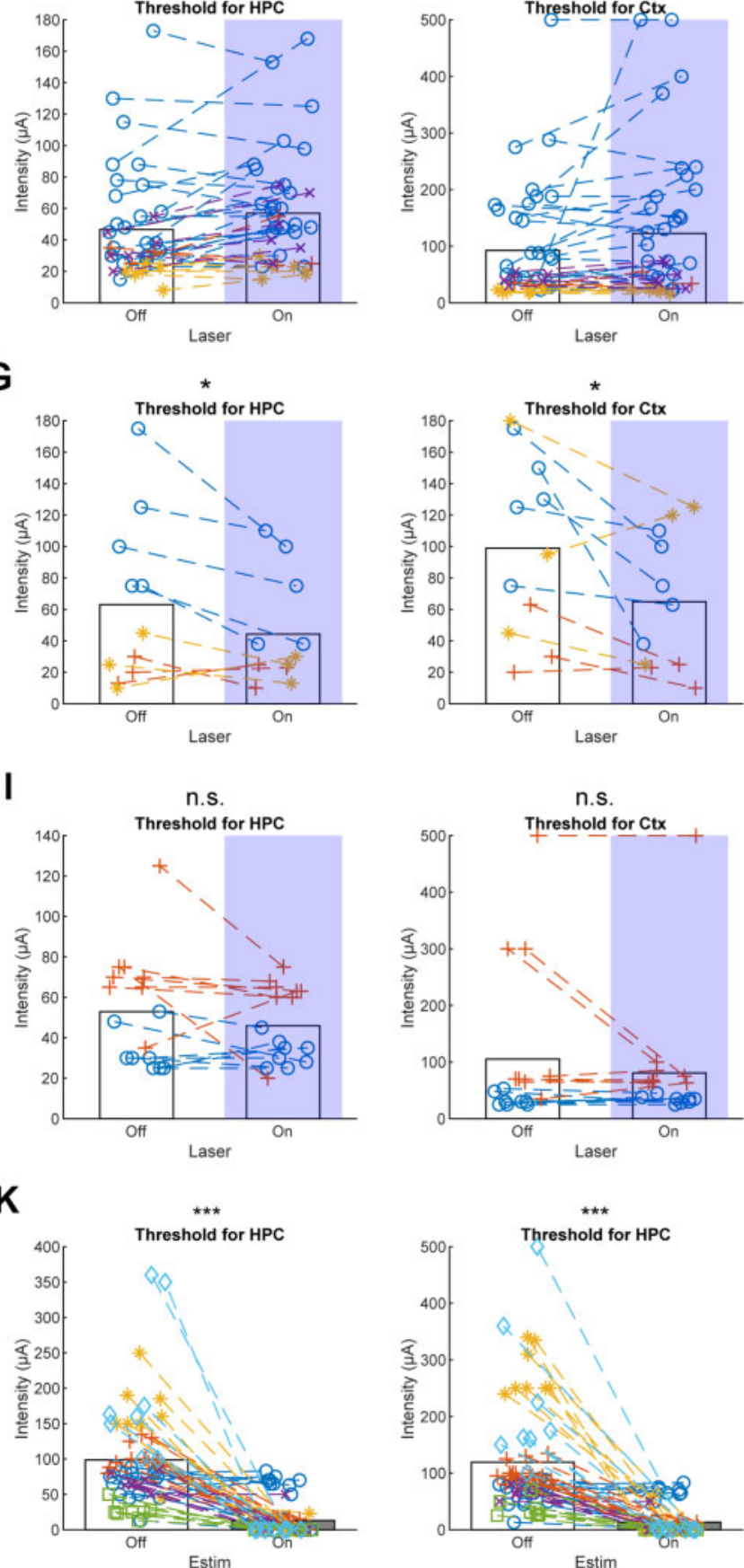

Figure 5 Medial septum cholinergic preconditioning decreases seizure susceptibility whereas medial septum E-stim preconditioning at seizure-like frequency increases it. (A) Preconditioning procedure. Medial septum neurons were optogenetically or electrically activated at $20 \mathrm{~Hz}$ for $60 \mathrm{~s}$ before seizure induction. (B) ChR2 signals were co-localized with cholinergic neurons in ChAT-Cre driver rats injected with a Cre-dependent ChR2-expressing viral vector. (C) cFos expression was co-localized with ChR2-expressing neurons in the rats after illumination. (D, $\mathbf{F}$ and $\mathbf{H}$ ) Schemata of the medial septum optogenetic preconditioning. Medial septum (MS) cholinergic, GABAergic or 
$P>0.05$; Supplementary Fig. 23F-H and J). The MI distribution was not skewed ( $P=0.39$, two-sample KolmogorovSmirnov test, Supplementary Fig. 23I). These results suggest that the phasic activation of medial septum cholinergic neurons during ictal periods is not effective in alleviating seizures of HPC origin.

\section{Medial septal cholinergic preconditioning decreases seizure susceptibility}

Finally, driven by our idea of preventing seizure development through prediction of ictal activity, we investigated whether the preactivation of any septal neuronal populations at seizure-like frequency decreased seizure susceptibility. We found earlier that medial septum cholinergic activation can decrease high-frequency HPC oscillations (Vandecasteele et al., 2014). Thus, we first activated medial septum cholinergic neurons in kindled rats at $20 \mathrm{~Hz}$ for $60 \mathrm{~s}$ before seizure induction (Fig. 5A and D). This preconditioning was repeated over several trials while the intensity of HPC commissure stimulation was progressively incremented to determine the threshold triggering HPC and Ctx seizures. Cholinergic preconditioning increased thresholds for HPC and Ctx seizures compared to those in non-illuminated trials $(46.6 \pm 34.1 \mu \mathrm{A}$ to $57.1 \pm 35.7 \mu \mathrm{A}$ and $92.7 \pm 99.5 \mu \mathrm{A}$ to $122.7 \pm 129.4 \mu \mathrm{A}$ per trial, $P<0.05$ for both, paired $t$-test; $36.0 \pm 16.4 \mu \mathrm{A}$ to $44.0 \pm 21.1 \mu \mathrm{A}$ and $56.3 \pm 50.5 \mu \mathrm{A}$ to $71.6 \pm 70.9 \mu \mathrm{A}$ per animal, $P=0.062$ and 0.231 , respectively; Fig. 5E). Five-second long preconditioning was not enough to decrease seizure susceptibility (data not shown). In contrast to cholinergic signalling, the preactivation of GABAergic neurons decreased thresholds for HPC and Ctx seizures compared to those in non-illuminated trials $(63.0 \pm 53.0 \mu \mathrm{A}$ to $44.3 \pm 34.6 \mu \mathrm{A}$ and $98.9 \pm 56.9 \mu \mathrm{A}$ to $64.9 \pm 43.2 \mu \mathrm{A}$ per trial, $P<0.05$ for both, paired $t$-test; $52.6 \pm 49.8 \mu \mathrm{A}$ to $38.1 \pm 29.6 \mu \mathrm{A}$ and $91.8 \pm 48.4 \mu \mathrm{A}$ to $62.2 \pm 37.6 \mu \mathrm{A}$ per animal, $P=0.340$ and 0.134 , respectively; Fig. $5 \mathrm{~F}$ and G). The preactivation of glutamatergic neurons did not cause any change in the thresholds $(52.9 \pm 27.4$ $\mu \mathrm{A}$ to $45.9 \pm 18.4 \mu \mathrm{A}$ and $105.1 \pm 137.6 \mu \mathrm{A}$ to $80.6 \pm 114.0$ $\mu \mathrm{A}$ per trial, $P>0.05$ for both, paired $t$-test; $52.9 \pm 27.8 \mu \mathrm{A}$ to $45.9 \pm 18.8 \mu \mathrm{A}$ and $105.1 \pm 101.6 \mu \mathrm{A}$ to $80.6 \pm 66.1 \mu \mathrm{A}$ per animal; Fig. $5 \mathrm{H}$ and I). To determine the net effects of simultaneous preactivation of these three neuronal populations, electrical preconditioning of the medial septum was then conducted (Fig. 5J). Medial septum E-stim at $20 \mathrm{~Hz}$ for $60 \mathrm{~s}$ before seizure induction significantly decreased thresholds for HPC and Ctx seizures $(98.6 \pm 67.3 \mu \mathrm{A}$ to $12.8 \pm 24.5 \mu \mathrm{A}$ and $119.3 \pm 96.9 \mu \mathrm{A}$ to $13.4 \pm 25.1 \mu \mathrm{A}$ per trial, $P<0.001$ for both, paired $t$-test; $103.0 \pm 63.0 \mu \mathrm{A}$ to $9.6 \pm 16.0 \mu \mathrm{A}$ and $129.7 \pm 99.3 \mu \mathrm{A}$ to $10.3 \pm 15.8 \mu \mathrm{A}$ per animal, $P<0.05$ for both; Fig. $5 \mathrm{~K})$. Notably, $20 \mathrm{~Hz}$ medial septum E-stim alone occasionally induced seizures during preconditioning exposure. These results suggest that the selective activation of medial septum cholinergic neurons during pre-ictal periods could prevent seizure development. However, this seizure-preventing effect is cell-type specific because the net effect of medial septum E-stim at $20 \mathrm{~Hz}$ (seizure-like frequency) is pro-seizure. Therefore, medial septum E-stim at seizure-like frequency should not be delivered during pre-ictal periods; instead it should be delivered during ictal periods in a closed-loop manner and precisely aligned to internal seizure patterns.

\section{Discussion}

We found that properly performed medial septum stimulation can terminate epileptic seizures of HPC origin and prevent their generalization. Notably, there was a dramatic difference between the outcomes of closed-loop and openloop interventions; closed-loop seizure rhythm-driven medial septum stimulation significantly shortened HPC seizures whereas open-loop stimulation did not shorten (and even prolonged) them. Considering the significant correlations between the modulation of HPC seizures and those of Ctx and motor seizures (Fig. 6), we claim bidirectional roles of the medial septum on TLE: anti- and pro-seizure effects (Fig. 7 and Supplementary Fig. 9). Our cell-type specific optogenetic experiments revealed that the medial septum GABAergic neuronal population mainly mediated the anti-seizure effects of the medial septum.

\section{Medial septum as a target of deep brain stimulation in refractory temporal lobe epilepsy: dual roles in anti-seizure effects}

The medial septum has dual anti-seizure effect roles: on-demand seizure-terminating effects and seizure susceptibilityreducing effects. Regarding the former, powerful on-demand seizure-terminating effects on already initiated episodes have not been reported with other proxy interventions in refractory TLE. E-stim of the anterior nucleus of the thalamus (ANT) (Fisher et al., 2010), the cortex (Morrell, 2011) and

Figure 5 Continued

glutamatergic neurons were selectively activated, respectively. (J) Schema of the medial septum electrical preconditioning. (E, G and I) Stimulus intensity thresholds for inducing HPC and Ctx seizures with or without preconditioning in medial septum cholinergic, GABAergic or glutamatergic neurons, respectively. (K) Stimulus intensity thresholds for inducing HPC and Ctx seizures with or without electrical preconditioning. $n=78$ trials from four rats (E), 22 trials from three rats (G), 32 trials from two rats (I) and 124 trials from six rats (K). Values are represented as columns for means, with a marker for each trial and a dedicated marker and colour for each rat. Statistical significance was tested using paired $t$ test. See also Supplementary Fig. 23. n.s. = not significant. $* P<0.05, * * P<0.01$, $* * * P<0.001$. 
A Open-loop responsive MS E-Stim (Sub-threshold seizure induction)
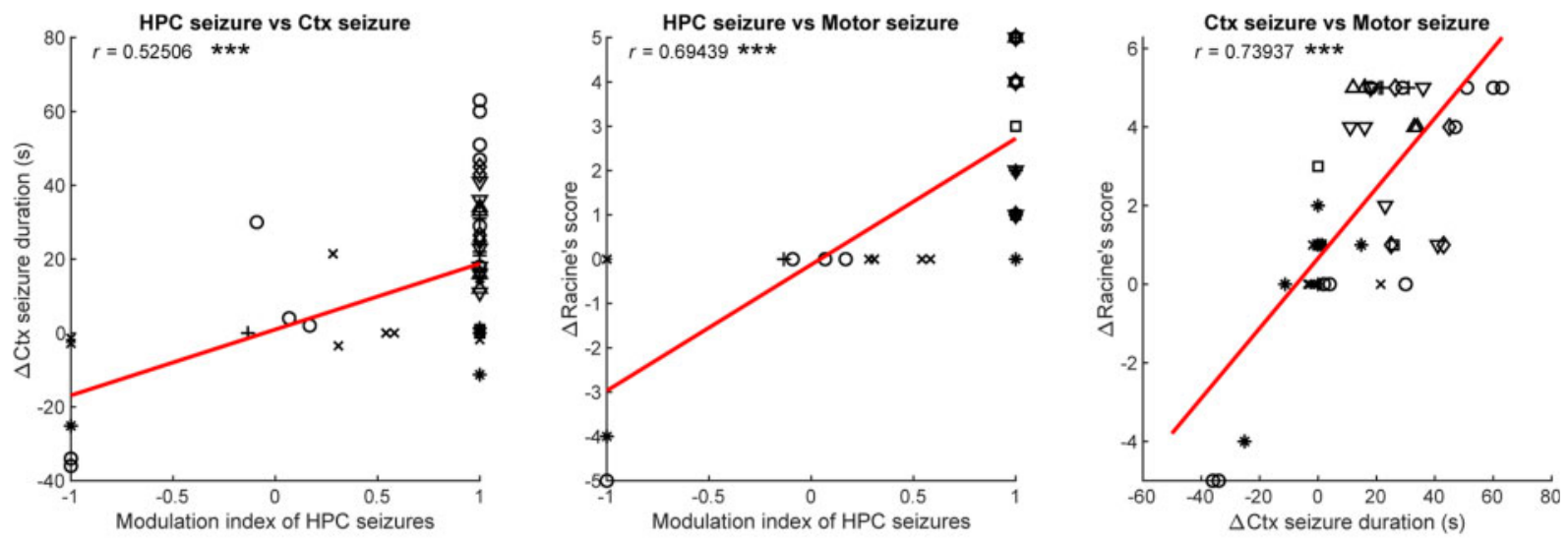

B Open-loop responsive MS E-Stim (Supra-threshold seizure induction)
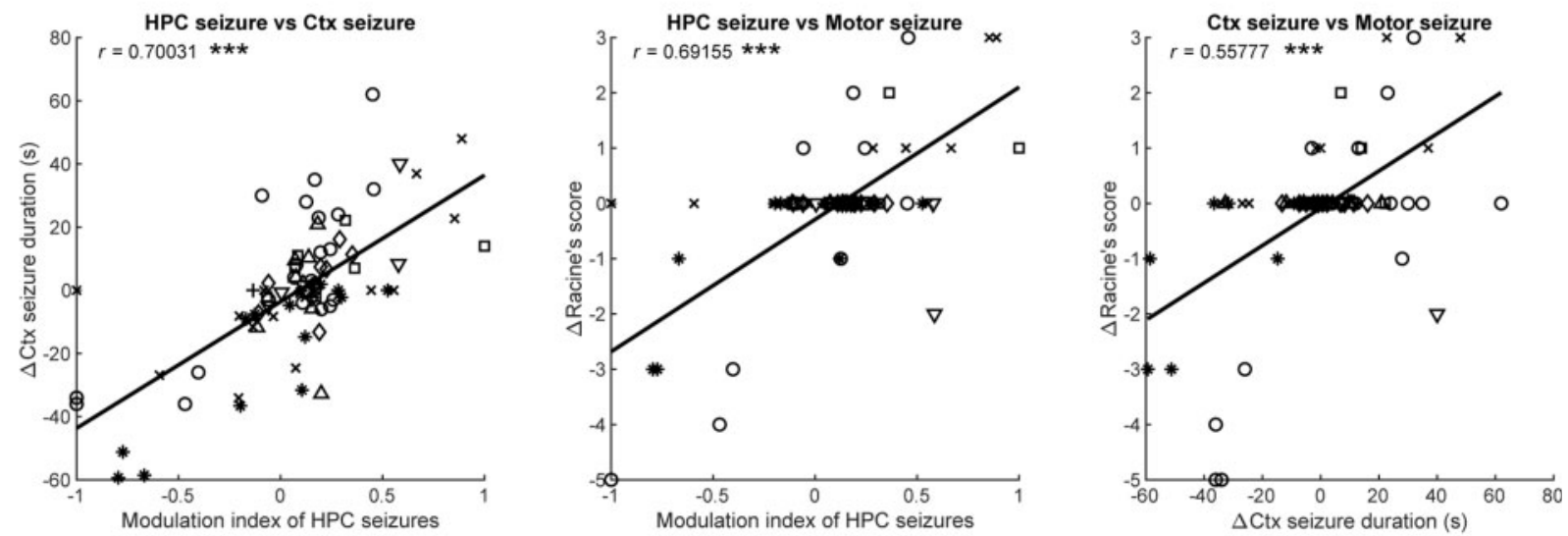

\section{Closed-loop seizure rhythm-driven MS E-Stim (Supra-threshold seizure induction)}
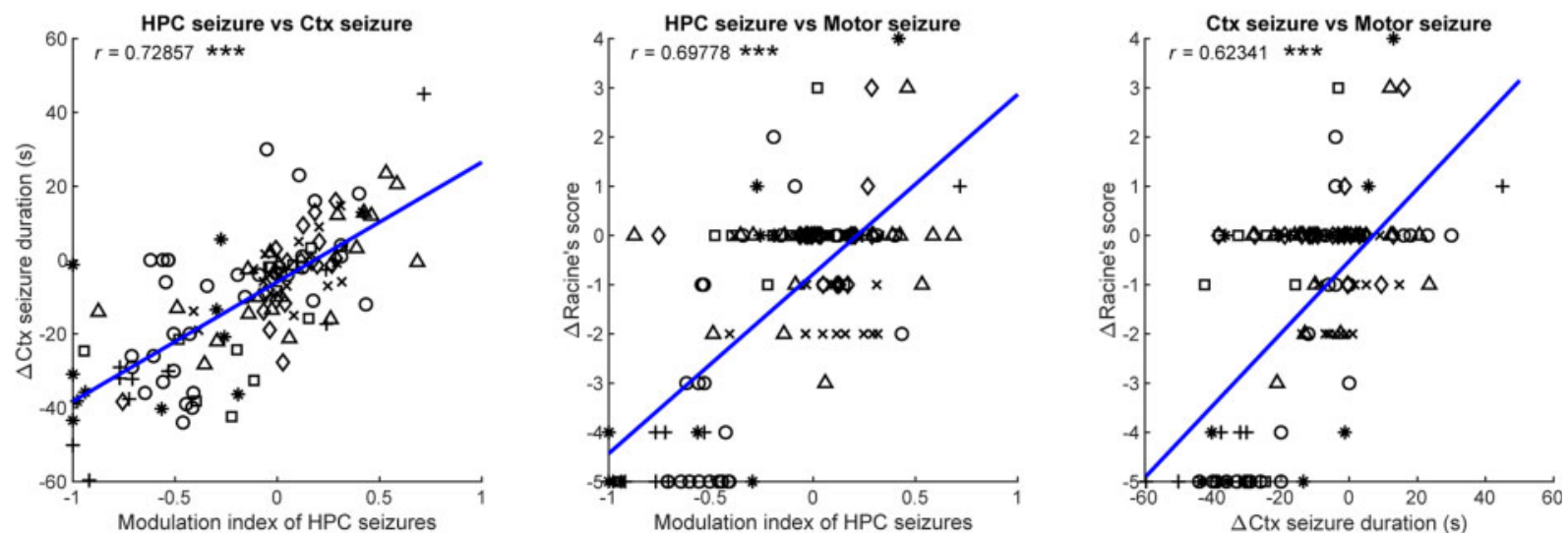

Figure 6 Modulations of HPC seizures correlate with the changes of Ctx electrographic and motor seizures. (A) Correlations between Mls of HPC seizure duration, changes of Ctx seizure durations and changes of motor seizure severity with or without open-loop responsive medial septum (MS) E-stim after subthreshold seizure inductions. Each marker represents a seizure episode. Lines indicate regression lines. $r=$ correlation coefficient. (B) The same conventions as in $\mathbf{A}$ but after suprathreshold seizure inductions. (C) The same conventions as in A but with or without closed-loop seizure rhythm-driven medial septum E-stim. Data in Figs 2 and 3 are replotted. Statistical significance was tested using Spearman's rank correlation.

the vagus nerve (Beekwilder and Beems, 2010) achieved $\sim 40-50 \%$ reduction in seizure occurrence in TLE patients. However, the remaining $50-60 \%$ of the episodes (which may become secondary generalized) remain to be treated. While existing treatments (including responsive neurostimulation) are decreasing seizure occurrence rather than 
A
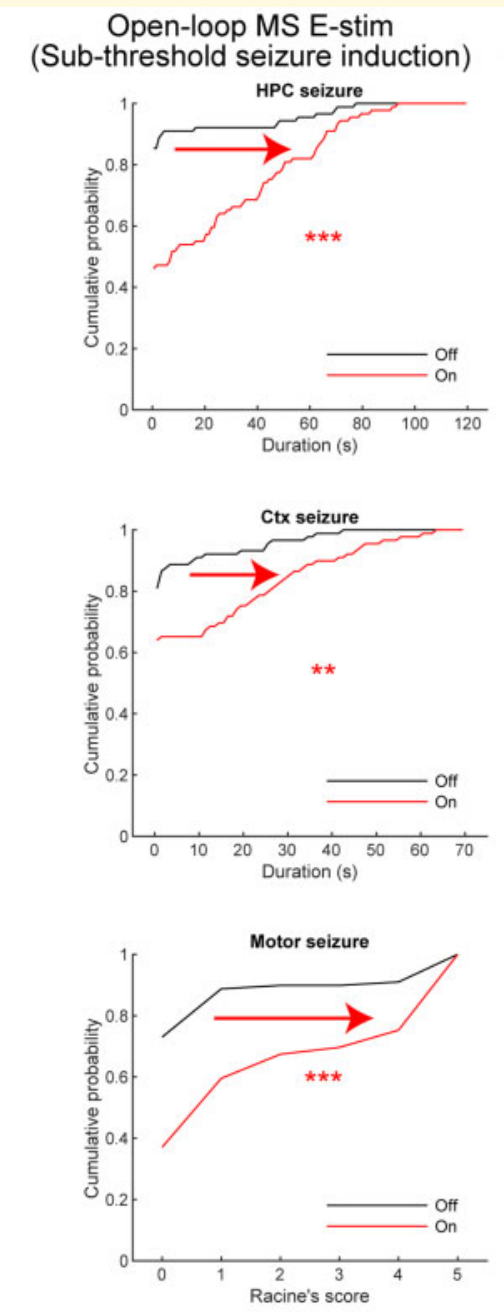

Open-loop MS E-stim

(Supra-threshold seizure induction)
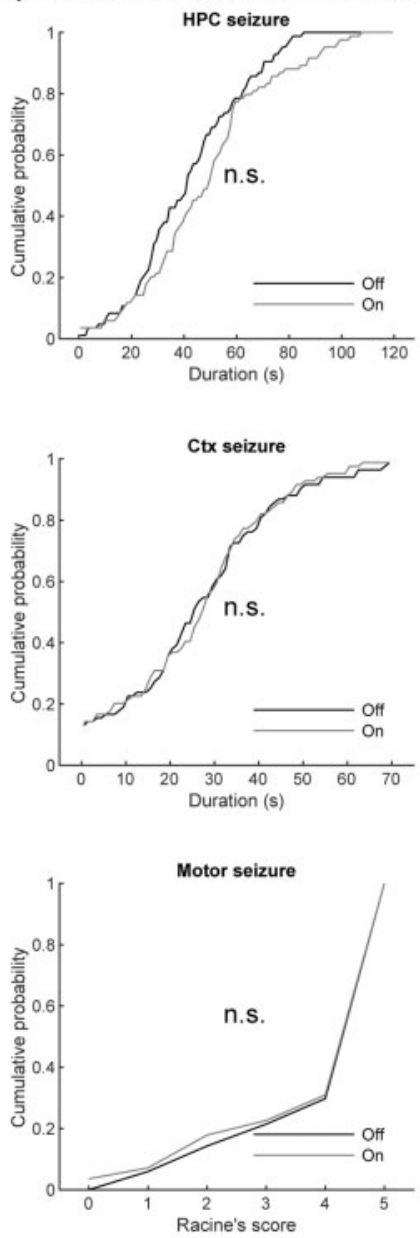

Closed-loop MS E-stim

(Supra-threshold seizure induction)
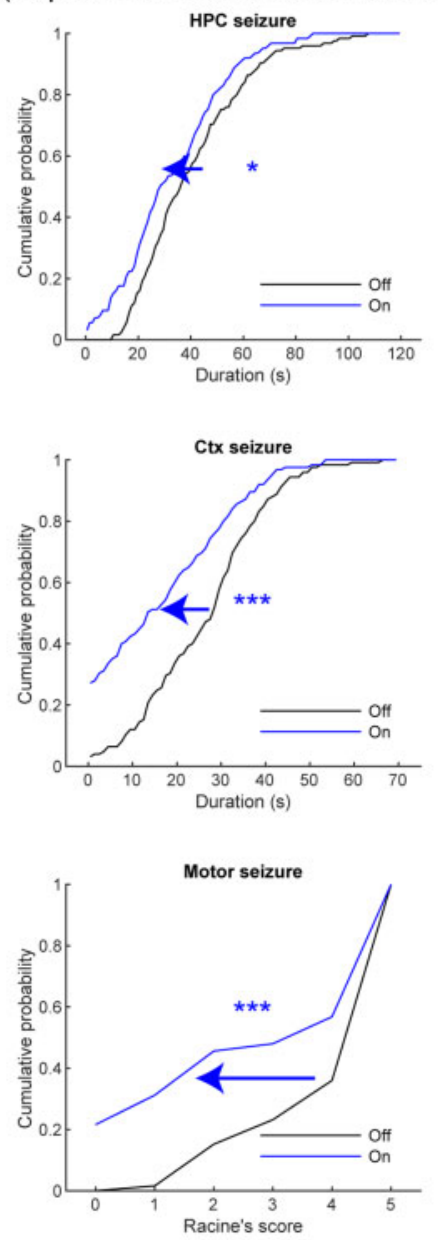

B

HPC electrographic seizures

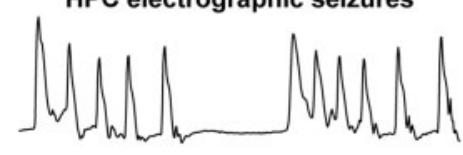

Hippocampus

Neocortex
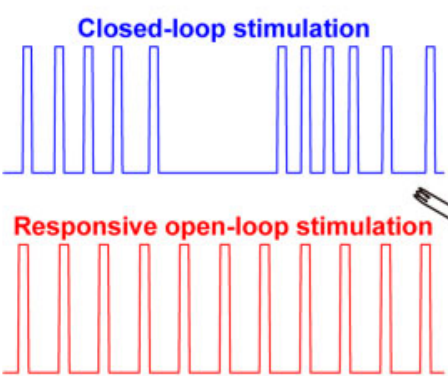

(-

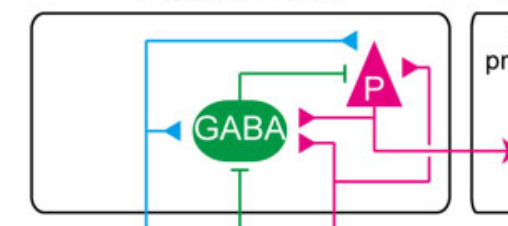

Seizure propagation

Anti-seizure effects (Closed-loop)
or

Pro-seizure effects (Open-loop)

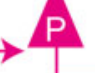

$\longrightarrow$ Motor seizures

Figure 7 Summary. (A) Pro- and anti-seizure effects of open- and closed-loop medial septum (MS) electrical stimulation. Data in Figs 2 and 3 are replotted. Statistical significance was analysed using two-sample Kolmogorov-Smirnov test. See also Supplementary Fig. 9. (B) Closed-loop seizure rhythm-driven medial septum E-stim was effective for terminating seizures of HPC origin and successfully suppressed secondary generalization. The precisely timed activation of medial septum GABAergic neurons may underlie the seizure-terminating effect. In contrast, responsive open-loop medial septum E-stim did not alter (or rather promoted) HPC seizures. ACh = cholinergic neurons; Glut = glutamategic neurons. 
shortening their durations (Morrell, 2011; Meiron et al., 2019), the closed-loop seizure rhythm driven medial septum stimulation was found to be able to terminate seizures even after their onsets (Fig. 3). The powerful seizure terminating effect of closed-loop medial septum E-stim presumably stems from the direct reciprocal connections of the medial septum and bilateral HPCs and the ECs (Müller and Remy, 2018; Tsanov, 2018), and from the precise timing of stimulation (Supplementary Fig. 12). This timing needs to be paced by the ongoing seizure rhythms for the entire seizure period (Supplementary Figs 5D and 6D). Regarding the other role of the medial septum (seizure susceptibility reducing effects), previous studies have shown that open-loop theta $(4-8 \mathrm{~Hz})$ medial septum E-stim reduces seizure susceptibility in animal models (Miller et al., 1994; Izadi et al., 2019) (note that open-loop medial septum E-stim modulates seizure susceptibility in a frequency dependent manner: $4-8 \mathrm{~Hz}$ reduces seizure susceptibility whereas $>20 \mathrm{~Hz}$ stimulation increases it, Fig. 5). This theta-rhythm medial septum stimulation has been thought to restore disrupted theta oscillations in the epileptic HPC presumably via cholinergic mechanisms (Colom et al., 2006; Soares et al., 2019). The dual anti-seizure effect roles of the medial septum make it a valuable DBS target for refractory TLE.

\section{Advantages of medial septum stimulation in temporal lobe epilepsy}

We point out three additional advantages of medial septum stimulation. First, because the medial septum is located at the midline, there is no need to insert multiple electrodes for bilateral neuromodulation. This is advantageous compared to the stimulation of the ANT, the HPC and the Ctx ( $\mathrm{Li}$ and Cook, 2018), because (for example) the ANTs are bilaterally located and each of them is only indirectly connected to the ipsilateral HPC via the Papez circuit (Paxinos and Watson, 2006). Second, electrical stimulation can be used for clinical practice: there is no need for optogenetics. Although the celltype specific seizure-terminating effects of the medial septum were revealed, only a seizure-rhythm-congruent stimulus delivery is practically required for seizure-terminating effects. Third, both seizure rhythm and theta rhythm stimulation can be delivered in an on-demand manner. This time-targeting (responsive) nature is a significant advantage over pharmaceutical treatments and traditional non-responsive brain stimulation (Morrell, 2011). Practically, non-responsive medial septum stimulation should be avoided so as not to disrupt cognitive functions supported by natural oscillations in the septohippocampal axis (Fuhrmann et al., 2015; Tsanov, 2017) and to prevent kindling effects. Repeated stimulus of the limbic system (including the medial septum) may make the circuit epileptic (Racine, 1972). Adaptive, but non-responsive DBS with real-time modulation of stimulus amplitude (Beudel and Brown, 2016) may not be suitable for the medial septum because even subthreshold stimuli may induce kindling effects. Responsive stimulus delivery to the medial septum will overcome the risk of kindling effects with non-responsive DBS. As current responsive neurostimulation devices have substantial false positive detections despite being tuned for individual patients, a closed-loop intervention driven exclusively by the ictal rhythms is required for medial septum stimulation to avoid possible kindling effects and undesired seizure generation. Together, combining responsive closed-loop ictal rhythm-driven seizure suppression with a seizure prediction based pre-ictal openloop theta rhythm seizure prevention may be a beneficial medial septum stimulation protocol to achieve successful TLE control.

\section{Possible mechanisms of seizure- terminating effects of closed-loop medial septum stimulation}

\section{Network mechanisms}

Three network mechanisms could underlie the seizure-terminating effects. The first is network stability. Seizure networks have multistable states: interictal, pre-ictal and ictal states (Jirsa et al., 2014; Kalitzin et al., 2019). Huge perturbation on network dynamics during ictal states would cause state transitions. Each medial septum stimulation may have only a small impact. However, if many stimuli are time-locked to a certain phase of seizure oscillations, either resonating with (in-phase) or occluding it (counter-phase), their impacts would be temporally summed (Supplementary Figs 5 and 6). The network dynamics could then be pushed away from the ictal state as a swing collapse. For example, the counterphase activation of medial septum or HPC projection neurons could occlude seizure oscillations with their refractory periods. In addition, the antidromic spikes of medial septum targeting HPC neurons presumably induce collisions on their axons and occlude seizure oscillations as well. Even excess in-phase positive feedback may disrupt the stability of seizure states like a howling phenomenon (Nordholm et al., 2018). However, open-loop stimulation would not cause such resonance because it was not phase-locked at all (the length of resultant vector $r$ was around 0.1; Supplementary Figs 5 and 6), and had a small impact on seizures (Fig. 2). The efficacy of closed-loop intervention varied trial-by-trial even in the same animal (Figs 3, 4 and Supplementary Fig. 15). This can stem from variability of the locked-phase angle (theta) and the phase-locking strength $(r)$ of medial septum intervention on HPC seizure waves (Supplementary Figs 5 and 6). Significantly, the phase-locking strength varied considerably during the first $20 \mathrm{~s}$ of seizures (Supplementary Figs $5 \mathrm{D}$ and $6 \mathrm{D}$ ). The distribution of phase-locking strengths for the first $20 \mathrm{~s}$ of seizures was bimodal (Supplementary Fig. 12B) and the coupling was significantly stronger in success trials than in non-success trials (Fig. $3 \mathrm{~F}$ and Supplementary Fig. 12A). More precisely timed stimulation leads to shorter HPC and Ctx seizure durations and less severe motor symptoms (Supplementary Fig. 12C and D). 
These results suggest that the binary-like outcome of closedloop intervention presumably depends on whether the detector of the closed-loop system can properly catch the seizure rhythm right at the beginning of seizures. In addition, pre-ictal brain states (Supplementary Fig. 11) may affect the rhythm recognition of the closed-loop intervention and impact outcomes (Ewell et al., 2015).

The second network mechanism that could underlie seizure-terminating effects of closed-loop medial septum stimulation is competition between different oscillation frequencies. Newly added oscillations influence pre-existing oscillations (Zutshi et al., 2018). Neuronal networks have their own resonating frequency and a closed-loop seizure-rhythm stimulation may induce a different oscillatory pattern occluding the generators of the original one. This could reduce the original oscillation.

The third network mechanism that could underlie seizuretermination is the bottleneck effect. Seizures of HPC origin reverberate and are amplified in both the HPC-HPC and the HPC-medial septum loops. To be secondary generalized, the EC is then passed through, which is a bottleneck structure in seizure spread. Medial septum GABAergic neurons project not only to the HPC but also to the EC in a very diffuse manner. The projection is primarily targeting interneurons in the EC as well as those in the HPC (Unal et al., 2015). EC and HPC interneurons have widespread hub-like oscillation gating effects there (Hájos and Paulsen, 2009; Cossart, 2014). These proxy and hub-like effects could then be synergistically amplified by the temporal summation of oscillation cycles to gate the EC signal.

\section{Cellular mechanisms}

The blue light illumination of medial septum cell populations in VGAT::ChR2, CaMKII $\alpha:$ ChR2, and ChAT::ChR2 rats induced very distinct spatiotemporal patterns of LFP deflection in the HPC and EC (Supplementary Fig. 1) and seizureterminating effects (Figs 4, 5 and Supplementary Figs 2123). Our failure to terminate seizures in CaMKII $\alpha:$ :ChR2 animals confirmed that the observed effects of our optogenetic experiments would not be artefacts of illumination or heating, which would happen even without any opsins (Owen et al., 2019). Instead, they were cell-type specific neuronal responses.

Medial septum GABAergic neurons project to the HPC and the EC and terminate mainly on GABAergic neurons (Freund and Antal, 1988). The phasic activation of medial septum GABAergic neurons would induce rebound firing of HPC GABAergic neurons. The rebound activation then introduces powerful peri-somatic inhibition on HPC pyramidal neurons around the next peak of seizure oscillations, and a synchronous population burst of pyramidal neurons occurs (Gulyás and Freund, 2015). This time-targeted inhibitory effect on pyramidal neurons can be temporally summed by their phase-locked nature and spatially amplified by widespread axonal innervation of the medial septum GABAergic neurons to the HPC and the EC (Unal et al., 2015). Because HPC GABAergic neurons are hypofunctional during TLE (which leads to the epileptiform discharges of pyramidal neurons) (Liu et al., 2014; Gulyás and Freund, 2015), this time-targeted phasic activation of HPC GABAergic neurons via the medial septum would be essential for terminating epileptic discharges in the HPC.

The on-demand seizure terminating effect is not grossly influenced by the septohippocampal cholinergic signalling because the latter is mainly mediated by slow metabotropic receptors (Desikan et al., 2018) that cannot follow the rhythmicity of seizure oscillations. Additional antiseizure effects of HPC cholinergic tone during ictal periods could not be observed. In contrast, the activation of medial septum cholinergic neurons in the pre-ictal periods reduced seizure susceptibility (Fig. 5). This is consistent with previous reports that activation of the medial septum cholinergic neurons decreased occurrence of high-frequency episodes in the HPC (Vandecasteele et al., 2014), which might even be mediated via GABAergic synaptic transmissions from the ChAT-positive medial septum neurons (Takács et al., 2018). Oriens-lacunosum moleculare cells in the HPC may mediate these anti-seizure effects (Haam et al., 2018).

The rapid, large LFP deflections in the HPC induced by the optogenetic activation of medial septum glutamatergic neurons confirmed the glutamatergic synaptic transmission (Supplementary Fig. 1). The medial septum-origin glutamatergic innervation of HPC excitatory and inhibitory neurons account for $7 \%$ and $21 \%$ of connection from the medial septum neurons, respectively (Sun et al., 2014). This means that glutamatergic inputs from the medial septum elicit both excitatory and inhibitory effects on the HPC network (Fig. 7). In addition, medial septum glutamatergic neurons have intense intraseptal synaptic connections, including with cholinergic neurons (Müller and Remy, 2018) that may have anti-seizure effects (Fig. 5). Therefore, the net effects of glutamatergic preconditioning on seizure susceptibility may become negligible via the sum of the compromising excitatory and inhibitory effects.

Our experiments revealed the seizure-terminating effect of closed-loop intervention in a provoked seizure model. The temporal organization of the stimulation may need to be adjusted if using other chronic, spontaneous seizure models since their underlying cellular and network mechanisms may be different. The feasibility of the medial septum-induced seizure termination in other seizure models shall be investigated in the future.

\section{Possible future clinical application}

Medial septum E-stim has been suggested for clinical studies after the finding that theta rhythm medial septum stimulation reduces seizure susceptibility (Fisher, 2015; Izadi et al., 2018). Together with the previous findings on open-loop stimulation of the medial septum (Miller et al., 1994; Izadi et al., 2019), our results indicate that a single electrode in the medial septum can be 
used for dual purposes: seizure termination via closedloop seizure rhythm-driven stimulation during ictal periods, and seizure prevention via open-loop theta rhythm stimulation during pre-ictal seizure-initiation periods. The development of reliable seizure prediction and detection algorithms for spontaneous seizures in humans would be a future challenge and is out of the scope of this work, as we only used a provoked seizure model in rats. The stability of closed-loop stimulation should be optimized by decreasing particularly false positive detections of stimulus artefacts and other ambient noises. Longitudinal, patient-specific fine-tuning of detection and stimulus algorithms would be important to ensure the long-term effectiveness and safety of medial septum E-stims. Intelligent DBS approaches utilizing deep learning technology may be effective for the real-time classification of brain states necessary for delivering proper stimulus patterns in an on-demand manner (Neumann et al., 2019).

In the present study, we showed that closed-loop seizure rhythm-driven medial septum E-stim successfully terminated HPC seizures and prevented their generalization and provided a concept for a novel therapeutic approach for TLE. The concept that precisely time-targeted proxy stimulation can produce large impacts on pathological oscillations could be extrapolated in the future to regulate other oscillopathies including movement or mental disorders (Takeuchi and Berényi, 2020).

\section{Acknowledgements}

We thank Ms Mari Takeuchi for technical assistance, Dr Karl Deisseroth for ChR2-expressing viral vectors and ChAT-Cre rats, and Dr Péter Hegyi for providing access to confocal microscopy.

\section{Funding}

This work was supported by KAKENHI (18KK0236, 19H03550, 19H05224), the Uehara Memorial Fund, the Kanae Foundation for the Promotion of Medical Science, and Life Science Foundation of Japan to Y.T., the Szeged Scientists Academy under the sponsorship of the Ministry of Human Capacities, Hungary (EMMI : 13725-2/2018/ INTFIN) to M.H., the UNKP-18-3 of the Ministry of Human Capacities, Hungary to G.K., the Momentum program II of the Hungarian Academy of Sciences, EFOP-3.6.116-2016-00008 and KKP133871/KKP20 grants of the National Research, Development and Innovation Office, Hungary, the 20391-3/2018/FEKUSTRAT of the Ministry of Human Capacities, Hungary, and the EU Horizon 2020 Research and Innovation Program (No. 739593 - HCEMM) to A.B.

\section{Competing interests}

A.B. is the owner of Amplipex Llc. and a shareholder of Neunos Ltd., Szeged, Hungary, manufacturers of signal-multiplexed neuronal amplifiers and neurostimulator devices.

\section{Supplementary material}

Supplementary material is available at Brain online.

\section{References}

Beekwilder JP, Beems T. Overview of the clinical applications of vagus nerve stimulation. J Clin Neurophysiol 2010; 27: 130-8.

Berens P. CircStat: a MATLAB Toolbox for circular statistics. J Statistical Software 2009; 31: 10.

Berényi A, Belluscio M, Mao D, Buzsáki G. Closed-loop control of epilepsy by transcranial electrical stimulation. Science 2012; 337: 735-7.

Berényi A, Somogyvári Z, Nagy AJ, Roux L, Long JD, Fujisawa S, et al. Large-scale, high-density (up to 512 channels) recording of local circuits in behaving animals. J Neurophysiol 2014; 111: 1132-49.

Berg AT, Vickrey BG, Langfitt JT, Sperling MR, Walczak TS, Shinnar S; Multicenter Study of Epilepsy Surgery, et al. The multicenter study of epilepsy surgery: recruitment and selection for surgery. Epilepsia 2003; 44: 1425-33.

Beudel M, Brown P. Adaptive deep brain stimulation in Parkinson's disease. Parkinsonism Relat Disord 2016; 22: S123-S6.

Bokil H, Andrews P, Kulkarni JE, Mehta S, Mitra PP. Chronux: a platform for analyzing neural signals. J Neurosci Methods 2010; 192: 146-51.

Bone B, Fogarasi A, Schulz R, Gyimesi C, Kalmar Z, Kovacs N, et al. Secondarily generalized seizures in temporal lobe epilepsy. Epilepsia 2012; 53: 817-24.

Buzsáki G. Theta oscillations in the hippocampus. Neuron 2002; 33: 325-40.

Chamberland S, Salesse C, Topolnik D, Topolnik L. Synapse-specific inhibitory control of hippocampal feedback inhibitory circuit. Front Cell Neurosci 2010; 4: 130.

Chen Z, Brodie MJ, Liew D, Kwan P. Treatment outcomes in patients with newly diagnosed epilepsy treated with established and new antiepileptic drugs: a 30-year longitudinal cohort study. JAMA Neurol 2018; 75: 279-86.

Colom LV, Castaneda MT, Reyna T, Hernandez S, Garrido-Sanabria E. Characterization of medial septal glutamatergic neurons and their projection to the hippocampus. Synapse 2005; 58: 151-64.

Colom LV, García-Hernández A, Castañeda MT, Perez-Cordova MG, Garrido-Sanabria ER. Septo-hippocampal networks in chronically epileptic rats: potential antiepileptic effects of theta rhythm generation. J Neurophysiol 2006; 95: 3645-53.

Cossart R. Operational hub cells: a morpho-physiologically diverse class of GABAergic neurons united by a common function. Curr Opin Neurobiol 2014; 26: 51-6.

Cukiert A, Lehtimäki K. Deep brain stimulation targeting in refractory epilepsy. Epilepsia 2017; 58: 80-4.

Dejean C, Courtin J, Karalis N, Chaudun F, Wurtz H, Bienvenu TCM, et al. Prefrontal neuronal assemblies temporally control fear behaviour. Nature 2016; 535: 420-4.

Desikan S, Koser DE, Neitz A, Monyer H. Target selectivity of septal cholinergic neurons in the medial and lateral entorhinal cortex. Proc Natl Acad Sci USA 2018; 115: E2644-E52.

Dutar P, Bassant MH, Senut MC, Lamour Y. The septohippocampal pathway: structure and function of a central cholinergic system. Physiol Rev 1995; 75: 393-427. 
Ewell LA, Liang L, Armstrong C, Soltesz I, Leutgeb S, Leutgeb JK. Brain state is a major factor in preseizure hippocampal network activity and influences success of seizure intervention. J Neurosci 2015; 35: 15635-48.

Fisher R, Salanova V, Witt T, Worth R, Henry T, Gross R; the SANTE Study Group, et al. Electrical stimulation of the anterior nucleus of thalamus for treatment of refractory epilepsy. Epilepsia 2010; 51: 899-908.

Fisher RS. Stimulation of the medial septum should benefit patients with temporal lobe epilepsy. Med Hypotheses 2015; 84: 543-50.

Fountas KN, Kapsalaki E, Hadjigeorgiou G. Cerebellar stimulation in the management of medically intractable epilepsy: a systematic and critical review. Foc 2010; 29: E8.

Freund TF, Antal M. GABA-containing neurons in the septum control inhibitory interneurons in the hippocampus. Nature 1988; 336: $170-3$.

Fuhrmann F, Justus D, Sosulina L, Kaneko H, Beutel T, Friedrichs D, et al. Locomotion, theta oscillations, and the speed-correlated firing of hippocampal neurons are controlled by a medial septal glutamatergic circuit. Neuron 2015; 86: 1253-64.

Fujisawa S, Amarasingham A, Harrison MT, Buzsáki G. Behavior-dependent short-term assembly dynamics in the medial prefrontal cortex. Nat Neurosci 2008; 11: 823-33.

Gelinas JN, Khodagholy D, Thesen T, Devinsky O, Buzsáki G. Interictal epileptiform discharges induce hippocampal-cortical coupling in temporal lobe epilepsy. Nat Med 2016; 22: 641-8.

Gritti I, Manns ID, Mainville L, Jones BE. Parvalbumin, calbindin, or calretinin in cortically projecting and GABAergic, cholinergic, or glutamatergic basal forebrain neurons of the rat. J Comp Neurol 2003; 458: 11-31.

Gulyás AI, Freund TT. Generation of physiological and pathological high frequency oscillations: the role of perisomatic inhibition in sharp-wave ripple and interictal spike generation. Curr Opin Neurobiol 2015; 31: 26-32.

Haam J, Zhou J, Cui G, Yakel JL. Septal cholinergic neurons gate hippocampal output to entorhinal cortex via oriens lacunosum moleculare interneurons. Proc Natl Acad Sci USA 2018; 115: E1886-95.

Hájos N, Paulsen O. Network mechanisms of gamma oscillations in the CA3 region of the hippocampus. Neural Networks 2009; 22: 1113-9.

Han C-L, Hu W, Stead M, Zhang T, Zhang J-G, Worrell GA, et al. Electrical stimulation of hippocampus for the treatment of refractory temporal lobe epilepsy. Brain Res Bull 2014; 109: 13-21.

Hazan L, Zugaro M, Buzsáki G, Klusters N. NDManager: a free software suite for neurophysiological data processing and visualization. J Neurosci Methods 2006; 155: 207-16.

Holmes MD, Miles AN, Dodrill CB, Ojemann GA, Wilensky AJ. Identifying potential surgical candidates in patients with evidence of bitemporal epilepsy. Epilepsia 2003; 44: 1075-9.

Iwasato T, Nomura R, Ando R, Ikeda T, Tanaka M, Itohara S. Dorsal telencephalon-specific expression of Cre recombinase in PAC transgenic mice. Genesis 2004; 38: 130-8.

Izadi A, Ondek K, Schedlbauer A, Keselman I, Shahlaie K, Gurkoff G. Clinically indicated electrical stimulation strategies to treat patients with medically refractory epilepsy. Epilepsia Open 2018; 3: 198-209.

Izadi A, Pevzner A, Lee DJ, Ekstrom AD, Shahlaie K, Gurkoff GG. Medial septal stimulation increases seizure threshold and improves cognition in epileptic rats. Brain Stimul 2019; 12: 735-42.

Jirsa VK, Stacey WC, Quilichini PP, Ivanov AI, Bernard C. On the nature of seizure dynamics. Brain 2014; 137: 2210-30.

Joshi A, Salib M, Viney T, Dupret D, Somogyi P. Behavior-dependent activity and synaptic organization of septo-hippocampal GABAergic neurons selectively targeting the hippocampal CA3 area. Neuron 2017; 96: 1342-57.

Kadir SN, Goodman DF, Harris KD. High-dimensional cluster analysis with the masked EM algorithm. Neural Comput 2014; 26: 2379-94.
Kalitzin S, Petkov G, Suffczynski P, Grigorovsky V, Bardakjian BL, Lopes da Silva F, et al. Epilepsy as a manifestation of a multistate network of oscillatory systems. Neurobiol Dis 2019; 130: 104488.

Kang D, Ding M, Topchiy I, Kocsis B. Reciprocal interactions between medial septum and hippocampus in theta generation: granger causality decomposition of mixed spike-field recordings. Front Neuroanat 2017; 11: 120.

Kozák G, Berényi A. Sustained efficacy of closed loop electrical stimulation for long-term treatment of absence epilepsy in rats. Sci Rep 2017; 7: 6300 .

Kozák G, Földi T, Berényi A. Chronic transcranial electrical stimulation and intracortical recording in rats. J Vis Exp 2018; 135: e56669.

Kwan P, Schachter SC, Brodie MJ. Drug-resistant epilepsy. N Engl J Med 2011; 365: 919-26.

Lerner-Natoli M, Rondouin G, Baldy-Moulinier M. Evolution of wet dog shakes during kindling in rats: comparison between hippocampal and amygdala kindling. Exp Neurol 1984; 83: 1-12.

Li MCH, Cook MJ. Deep brain stimulation for drug-resistant epilepsy. Epilepsia 2018; 59: 273-90.

Liu Y-Q, Yu F, Liu W-H, He X-H, Peng B-W. Dysfunction of hippocampal interneurons in epilepsy. Neurosci Bull 2014; 30: 985-98.

Malow BA, Carney PR, Kushwaha R, Bowes RJ. Hippocampal sleep spindles revisited: physiologic or epileptic activity? Clin Neurophysiol 1999; 110: 687-93.

Massey CA, Sowers LP, Dlouhy BJ, Richerson GB. Mechanisms of sudden unexpected death in epilepsy: the pathway to prevention. Nat Rev Neurol 2014; 10: 271-82.

McIntyre DC, Gilby KL. Mapping seizure pathways in the temporal lobe. Epilepsia 2008; 49: 23-30.

Meiron O, Gale R, Namestnic J, Bennet-Back O, Gebodh N, Esmaeilpour Z, et al. Antiepileptic effects of a novel non-invasive neuromodulation treatment in a subject with early-onset epileptic encephalopathy: case report with 20 sessions of HD-tDCS intervention. Front Neurosci 2019; 13: 547.

Miller JW, Turner GM, Gray BC. Anticonvulsant effects of the experimental induction of hippocampal theta activity. Epilepsy Res 1994; 18: 195-204.

Monai H, Ohkura M, Tanaka M, Oe Y, Konno A, Hirai H, et al. Calcium imaging reveals glial involvement in transcranial direct current stimulation-induced plasticity in mouse brain. Nat Commun 2016; 7: 11100.

Morrell MJ; On behalf of the RNS System in Epilepsy Study Group. Responsive cortical stimulation for the treatment of medically intractable partial epilepsy. Neurology 2011; 77: 1295-304.

Moxon KA, Shahlaie K, Girgis F, Saez I, Kennedy J, Gurkoff GG. From adagio to allegretto: the changing tempo of theta frequencies in epilepsy and its relation to interneuron function. Neurobiol Dis 2019; 129: 169-81.

Müller C, Remy S. Septo-hippocampal interaction. Cell Tissue Res 2018; 373: 565-75.

Neumann W-J, Turner RS, Blankertz B, Mitchell T, Kühn AA, Richardson MR. Toward electrophysiology-based intelligent adaptive deep brain stimulation for movement disorders Neurotherapeutics 2019; 16: 105-18.

Nordholm S, Schepker H, Tran LTT, Doclo S. Stability-controlled hybrid adaptive feedback cancellation scheme for hearing aids. J Acoust Soc Am 2018; 143: 150-66.

Owen SF, Liu MH, Kreitzer AC. Thermal constraints on in vivo optogenetic manipulations. Nat Neurosci 2019; 22: 1061-5.

Paxinos G, Watson C. The rat brain in stereotaxic coordinates. 6th ed. New York: Academic Press; 2006.

Paz JT, Huguenard JR. Microcircuits and their interactions in epilepsy: is the focus out of focus? Nat Neurosci 2015; 18: 351-9.

Racine RJ. Modification of seizure activity by electrical stimulation. II. Motor seizure. Electroencephalogr Clin Neurophysiol 1972; 32: 281-94. 
Robinson J, Manseau F, Ducharme G, Amilhon B, Vigneault E, El Mestikawy S, et al. Optogenetic activation of septal glutamatergic neurons drive hippocampal theta rhythms. J Neurosci 2016; 36: 3016-23.

Rossant C, Kadir SN, Goodman DF, Schulman J, Hunter ML, Saleem $\mathrm{AB}$, et al. Spike sorting for large, dense electrode arrays. Nat Neurosci 2016; 19: 634-41.

Royer S, Zemelman BV, Barbic M, Losonczy A, Buzsáki G, Magee JC. Multi-array silicon probes with integrated optical fibers: lightassisted perturbation and recording of local neural circuits in the behaving animal. Eur J Neurosci 2010; 31: 2279-91.

Selwa LM, Schmidt SL, Malow BA, Beydoun A. Long-term outcome of nonsurgical candidates with medically refractory localizationrelated epilepsy. Epilepsia 2003; 44: 1568-72.

Sinel'nikova VV, Popova YI, Kichigina VF. Correlational relationships between the hippocampus and medial septal area and their changes during epileptogenesis. Neurosci Behav Physi 2009; 39: 619-23.

Soares JI, Costa C, Ferreira MH, Andrade PA, Maia GH, Lukoyanov NV. Partial depletion of septohippocampal cholinergic cells reduces seizure susceptibility, but does not mitigate hippocampal neurodegeneration in the kainate model of epilepsy. Brain Res 2019; 1717: $235-46$.

Sun Y, Nguyen AQ, Nguyen JP, Le L, Saur D, Choi J, et al. Cell-typespecific circuit connectivity of hippocampal CA1 revealed through Cre-dependent rabies tracing. Cell Rep 2014; 7: 269-80.

Takács VT, Cserép C, Schlingloff D, Pósfai B, Szőnyi A, Sos KE, et al. Co-transmission of acetylcholine and GABA regulates hippocampal states. Nat Commun 2018; 9: 2848.
Takeuchi Y, Berényi A. Oscillotherapeutics - Time-targeted interventions in epilepsy and beyond. Neuroscience Research 2020; 152: 87-107.

Tsanov M. Speed and oscillations: medial septum integration of attention and navigation. Front Syst Neurosci 2017; 11:

Tsanov M. Differential and complementary roles of medial and lateral septum in the orchestration of limbic oscillations and signal integration. Eur J Neurosci 2018; 48: 2783-94.

Unal G, Joshi A, Viney TJ, Kis V, Somogyi P. Synaptic targets of medial septal projections in the hippocampus and extrahippocampal cortices of the mouse. J Neurosci 2015; 35: 15812-26.

Vandecasteele M, Varga V, Berényi A, Papp E, Barthó P, Venance L, et al. Optogenetic activation of septal cholinergic neurons suppresses sharp wave ripples and enhances theta oscillations in the hippocampus. Proc Natl Acad Sci USA 2014; 111: 13535-40.

Wang Y, Liang J, Xu C, Wang Y, Kuang Y, Xu Z, et al. Low-frequency stimulation in anterior nucleus of thalamus alleviates kainate-induced chronic epilepsy and modulates the hippocampal EEG rhythm. Exp Neurol 2016; 276: 22-30.

Witten IB, Steinberg EE, Lee SY, Davidson TJ, Zalocusky KA, Brodsky M, et al. Recombinase-driver rat lines: tools, techniques, and optogenetic application to dopamine-mediated reinforcement. Neuron 2011; 72: 721-33.

Zutshi I, Brandon MP, Fu ML, Donegan ML, Leutgeb JK, Leutgeb S. Hippocampal neural circuits respond to optogenetic pacing of theta frequencies by generating accelerated oscillation frequencies. Curr Biol 2018; 28: 1179-88. 